\title{
PHYSICAL STRUCTURE OF THE PROTO-PLANETARY NEBULA CRL 618. I. OPTICAL LONG-SLIT SPECTROSCOPY AND IMAGING
}

\author{
C. Sánchez Contreras and R. Sahai \\ Jet Propulsion Laboratory, California Institute of Technology, MS 183-900, 4800 Oak Grove Drive, Pasadena, CA 91109; \\ sanchez@eclipse.jpl.nasa.gov, sahai@eclipse.jpl.nasa.gov \\ AND \\ A. GIL DE PAZ \\ NASA/IPAC Extragalactic Database, California Institute of Technology, MS 100-22, Pasadena, CA 91125; \\ agpaz@ipac.caltech.edu \\ Received 2002 April 18; accepted 2002 June 13
}

\begin{abstract}
In this paper we present optical long-slit spectroscopy and imaging of the proto-planetary nebula CRL 618. The optical lobes of CRL 618 consist of shock-excited gas, which emits many recombination and forbidden lines, and dust, which scatters light from the innermost regions. From the analysis of the scattered $\mathrm{H} \alpha$ emission, we derive a nebular inclination of $i=24^{\circ} \pm 6^{\circ}$. The spectrum of the innermost part of the east lobe (visible as a bright, compact nebulosity close to the star in the H $\alpha$ Hubble Space Telescope image) is remarkably different from that of the shocked lobes but similar to that of the inner $\mathrm{H}$ II region, suggesting that this region represents the outermost parts of the latter. We find a nonlinear radial variation of the gas velocity along the lobes. The largest projected LSR velocities (with respect to the systemic velocity), $\sim 80 \mathrm{~km} \mathrm{~s}^{-1}$, are measured at the tips of the lobes, where the direct images show the presence of compact bow-shaped structures. The velocity of the shocks in CRL 618 is in the range $\sim 75-200 \mathrm{~km} \mathrm{~s}^{-1}$, as derived from diagnostic line ratios and line profiles. We report a brightening (weakening) of [O III] $\lambda 5007$ ([O I] $\lambda 6300$ ) over the last $\sim 10$ years that may indicate a recent increase in the speed of the exciting shocks. From the analysis of the spatial variation of the nebular extinction, we find a large density contrast between the material inside the lobes and beyond them: the optical lobes seem to be "cavities" excavated in the asymptotic giant branch (AGB) envelope by interaction with a more tenuous post-AGB wind. The electron density, with a mean value $n_{e} \sim 5 \times 10^{3}-10^{4} \mathrm{~cm}^{-3}$, shows significant fluctuations but no systematic decrease along the lobes, in agreement with most line emission arising in a thin shell of shocked material (the lobe walls) rather than in the post-AGB wind filling the interior of the lobes. The masses of atomic and ionized gas, respectively, in the east (west) lobe are $>1.3 \times 10^{-4}\left(>7 \times 10^{-5}\right)$ and $\sim 6 \times 10^{-5}\left(\sim 4 \times 10^{-5}\right) M_{\odot}$. The shocks in CRL 618 are in a radiative regime and may lead in the future to the evolution of the optically emitting lobes into a fast, bipolar molecular outflow. The time required by the dense, shocked gas to cool down significantly is $\lesssim 2 \mathrm{yr}$, which is substantially lower than the kinematical age of the lobes $(\lesssim 180 \mathrm{yr})$. This result suggests that a fast wind is currently active in CRL 618 and keeps shocking the circumstellar material.
\end{abstract}

Subject headings: circumstellar matter — planetary nebulae: individual (CRL 618) - reflection nebulae stars: AGB and post-AGB — stars: mass loss — stars: winds, outflows

On-line material: color figures

\section{INTRODUCTION}

The physical mechanisms responsible for the onset of bipolarity and polar acceleration in planetary nebulae (PNs) are already active in the first stages of the evolution beyond the asymptotic giant branch (AGB), i.e., in protoplanetary nebulae (PPNs, also called post-AGB objects). Therefore, PPNs and young PNs hold the key for understanding the complex and fast $\left(\sim 10^{3} \mathrm{yr}\right)$ nebular evolution from the AGB toward the PN phase. Such evolution is believed (by an increasing number of astronomers) to be governed by the interaction between fast, collimated winds or jets, ejected in the late AGB or early post-AGB phase, and the spherical and slowly expanding circumstellar envelope (CSE) resulting from the star mass-loss process during the AGB (see Sahai \& Trauger 1998; Kastner, Soker, \& Rappaport 2000).

CRL 618 (=RAFGL 618=IRAS 04395+3601=Westbrook Nebula) is a well-studied PPN that has very recently started its post-AGB journey (only $\sim 200$ years ago; e.g., Kwok \& Bignell 1984) and is rapidly evolving toward the PN stage. Most of the circumstellar matter in CRL 618 is still in the form of molecular gas. This component, with a total mass of $\approx 1.5 M_{\odot}$, consists of (1) a spherical and extended $\left(\gtrsim 20^{\prime \prime}\right)$ envelope expanding at $17.5 \mathrm{~km} \mathrm{~s}^{-1}$ (Knapp \& Morris 1985; Bujarrabal et al. 1988, 2001; Bachiller et al. 1988; Hajian, Phillips, \& Terzian 1996; Phillips et al. 1992) and (2) an inner, compact bipolar outflow moving away from the star at velocities $\gtrsim 200 \mathrm{~km} \mathrm{~s}^{-1}$ (Cernicharo et al. 1989; Gammie et al. 1989; Meixner et al. 1998; Neri et al. 1992). The outer, slowly expanding component is interpreted as the result from the mass-loss process of the central star during the AGB, which took place at a rate of $\dot{M}=$ few times $10^{-5}$ to $10^{-4} M_{\odot} \mathrm{yr}^{-1}$. The fast bipolar outflow, with a mass of $\sim 10^{-2} M_{\odot}$, is believed to be the result of the interaction between a fast, collimated post-AGB wind and the spherical AGB CSE (see references above). 
The high-excitation nebula (atomic and ionized gas) is composed of (1) a compact $\mathrm{H}$ II region, visible through radio continuum emission, elongated in the east-west direction with an angular size of $0.2-0$ ". 4 that is increasing with time (Kwok \& Feldman 1981; Martín-Pintado et al. 1993), and (2) multiple lobes with shock-excited gas that produces recombination and forbidden line emission in the optical (e.g., Goodrich 1991; Trammell, Dinerstein, \& Goodrich 1993; Kelly, Latter, \& Rieke 1992; Trammell 2000). From previous spectroscopic data, the inner $\mathrm{H}$ II region and the lobes are known to be expanding with velocities of $\sim 20$ and up to $\sim 120 \mathrm{~km} \mathrm{~s}^{-1}$, respectively (Martín-Pintado et al. 1988; Carsenty \& Solf 1982, hereafter CS82). The analysis of different optical line ratios indicates that a relatively large range of temperatures $(\approx 10,000-25,000 \mathrm{~K})$ and densities $\left(\approx 1600-8000 \mathrm{~cm}^{-3}\right)$ are present in the lobes (Kelly et al. 1992).

The optical spectrum of CRL 618 also shows a weak, red continuum that is the stellar light reflected by the nebular dust. From spectropolarimetric observations it is known that a fraction of the flux of the Balmer lines is also scattered light originally arising from the inner, compact $\mathrm{H}$ II region (Schmidt \& Cohen 1981; Trammell et al. 1993). The polarization of the forbidden lines is negligible, indicating that they are almost entirely produced by the shock-excited gas in the lobes with a small or insignificant contribution by scattered photons from the $\mathrm{H}$ II region (the high density in this region, $\sim 10^{6} \mathrm{~cm}^{-3}$, produces collisional de-excitation of most forbidden lines).

The central star of CRL 618 has been classified as B0 based on the shape of the dereddened visual continuum (Schmidt \& Cohen 1981) and on the weak [O III] line emission from the inner, compact $\mathrm{H}$ II region (Kaler 1978). The luminosity of CRL 618, obtained by integrating the observed IRAS fluxes, is $L=1.22 \times 10^{4} \quad L_{\odot}(D / \mathrm{kpc})^{2}$ (Goodrich 1991). Based on the $L$-values predicted by evolutionary models $\left(\sim 10^{4} L_{\odot}\right)$ and the typical scale height of PNs $(\sim 120 \mathrm{pc})$, these authors calculate a distance to the source of $0.9 \mathrm{kpc}$, which we also assume in this paper.

In this paper (Paper I of a series of two) we report optical imaging and long-slit spectroscopy of CRL 618. Observational techniques and data reduction are described in $\S 2$. In $\S \S 3$ and 4 we present our observations, including a brief description of the nebular morphology and main characteristics of the optical spectrum. In $\S 5$ we analyze the different emission-line components and study the physical properties of the different nebular regions probed by them. In $\S \S 6$ and 7 we derive the mean nebular inclination and describe the kinematics of CRL 618, respectively. The spatial distribution along the nebular axis of the extinction and the electron density are presented, respectively, in $\delta \S 8$ and 9 . In the latter, we also estimate the atomic and ionized mass in different regions. Finally, we discuss our results and give a possible scenario to explain the formation and future evolution of CRL 618 in $\S 10$. The main conclusions of this work are summarized in $\S 11$.

\section{OBSERVATIONS AND DATA REDUCTION}

\subsection{Optical Imaging}

\subsubsection{Ground-based}

We have obtained narrowband images of the $\mathrm{H} \alpha$ line and the adjacent continuum emission in the PPN CRL 618
(Figs. $1 a$ and $1 b$ ). Observations were carried out on 2001 November 8 and 9 with the Palomar Observatory 60 inch $(1.5 \mathrm{~m})$ telescope. The detector was the CCD camera 13, which has $2048 \times 2048$ pixels of $24 \mu \mathrm{m}$ in size and provides a plate scale of 0 "! 378 pixel $^{-1}$. The narrowband filters used for imaging the $\mathrm{H} \alpha$ line and adjacent continuum are centered at 6563 and $6616 \AA$, respectively, and have an FWHM of $20 \AA$. Weather conditions during the observations were good, although nonphotometric, and the seeing ranged between 0.9 and $2^{\prime \prime}$. The final spatial resolution of the $\mathrm{H} \alpha$ (continuum) combined images is 1 !'9 (1".1). Total exposure times are $2700 \mathrm{~s}$ for the $\mathrm{H} \alpha$ image and $6300 \mathrm{~s}$ for the continuum.

Data reduction, including bias subtraction, flat-field correction, removal of cosmic rays and bad pixels, and registering plus combination of individual images, was performed following standard procedures within IRAF. ${ }^{1}$

Astrometry of our images was done cross-correlating the coordinates of 376 and 187 field stars (for $\mathrm{H} \alpha$ and the continuum, respectively) in the CCD and those in the USNOA2.0 catalog. This procedure yields a plate scale of 0 ".378 \pm 0 ".009 pixel $^{-1}$. The coordinates of the origin of our spatial scale in our images are R.A. $=04^{\mathrm{h}} 42^{\mathrm{m}} 53^{\mathrm{s}} .55$ and decl. $=36^{\circ} 06^{\prime} 54^{\prime \prime} 5(\mathrm{~J} 2000.0)$. The errors of the absolute positions derived are $\lesssim 0$ ". 4 (considering the standard deviation of our best calibration solution, 0 ". 15 , and the absolute position error of the USNO stars, about 0 ". 25 ).

We have obtained a pure $\mathrm{H} \alpha$ line emission image by subtracting the continuum emission to our original $\mathrm{H} \alpha$ plus continuum image. The continuum image was first smoothed to match the lower effective spatial resolution of the $\mathrm{H} \alpha$ image, and then the flux of several field stars was measured in both images to derive a scaling factor that includes the differences of the system " filter+CCD" response between the two images as well as the different exposure times and sky transparency when the observations were performed.

Flux calibration was performed using the USNO-A2.0 $R$ magnitudes of the field stars. The error in the flux calibration is dominated by the absolute flux error in the USNOA2.0 catalog $(\sim 55 \%)$. The flux calibration of the $\mathrm{H} \alpha$ and continuum images is consistent with our calibrated long-slit spectra within a factor of $\lesssim 2$.

\subsubsection{Hubble Space Telescope}

As a complement to our data, we have used the high angular resolution $\mathrm{H} \alpha$ image of CRL 618 from the Hubble Space Telescope (HST) archive (GO 6761; PI S. Trammell). This image was obtained with the Wide Field Planetary Camera 2 (WFPC2) and the narrowband filter F656N, around the $\mathrm{H} \alpha$ line. The WFPC2 has a $36^{\prime \prime} \times 36^{\prime \prime}$ field of view and a plate scale of 0.0455 pixel $^{-1}$. The image (Fig. 1c) is pipeline reduced.

\subsection{Optical Long-Slit Spectroscopy}

We obtained optical long-slit spectra of CRL 618 on 2000 November 12 and 13, using the Intermediate Dispersion Spectrograph (IDS) of the $2.5 \mathrm{~m}$ Isaac Newton Telescope of the Roque de los Muchachos Observatory (La Palma, Spain). The detector was the EEV10 CCD, with squared

\footnotetext{
${ }^{1}$ IRAF is distributed by the National Optical Astronomy Observatory, which is operated by the Association of Universities for Research in Astronomy, Inc., under cooperative agreement with the National Science Foundation.
} 


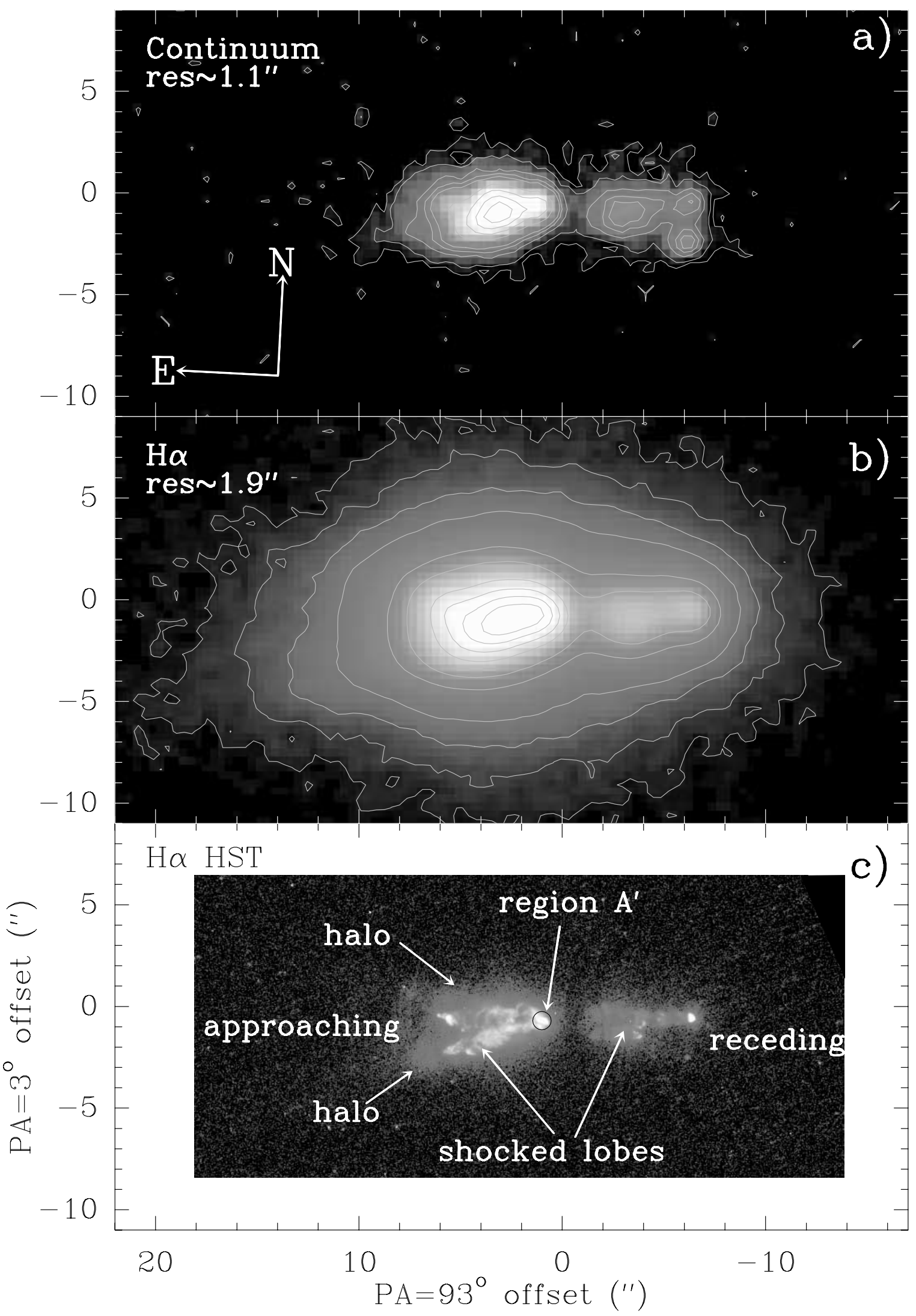

FIG. 1.-Direct imaging of the PPN CRL 618. (a) Narrowband, ground-based image of the continuum emission at $6616 \AA$. Contours are $3 \sigma, 5 \sigma$, $10 \sigma, 15 \sigma$, and from $20 \sigma$ to $120 \sigma$ by $20 \sigma$, with $\sigma=7 \times 10^{-19} \mathrm{ergs} \mathrm{s}^{-1} \mathrm{~cm}^{-2} \AA^{-1} \operatorname{arcsec}^{-2}$. (b) Narrowband, ground-based image of the H $\alpha$ emission line (continuum subtracted). Contours are $5 \sigma, 10 \sigma, 20 \sigma, 40 \sigma, 150 \sigma$, and from $300 \sigma$ to $2300 \sigma$ by $500 \sigma$, with $\sigma=6.5 \times 10^{-17} \mathrm{ergs} \mathrm{s}^{-1} \mathrm{~cm}^{-2} \mathrm{arcsec}^{-2}$. (c) Narrowband WFPC2/HST image in the light of $\mathrm{H} \alpha$ plus continuum. The continuum contribution in this image is less than $\sim 3$ in any region. [See the electronic edition of the Journal for a color version of this figure.] 


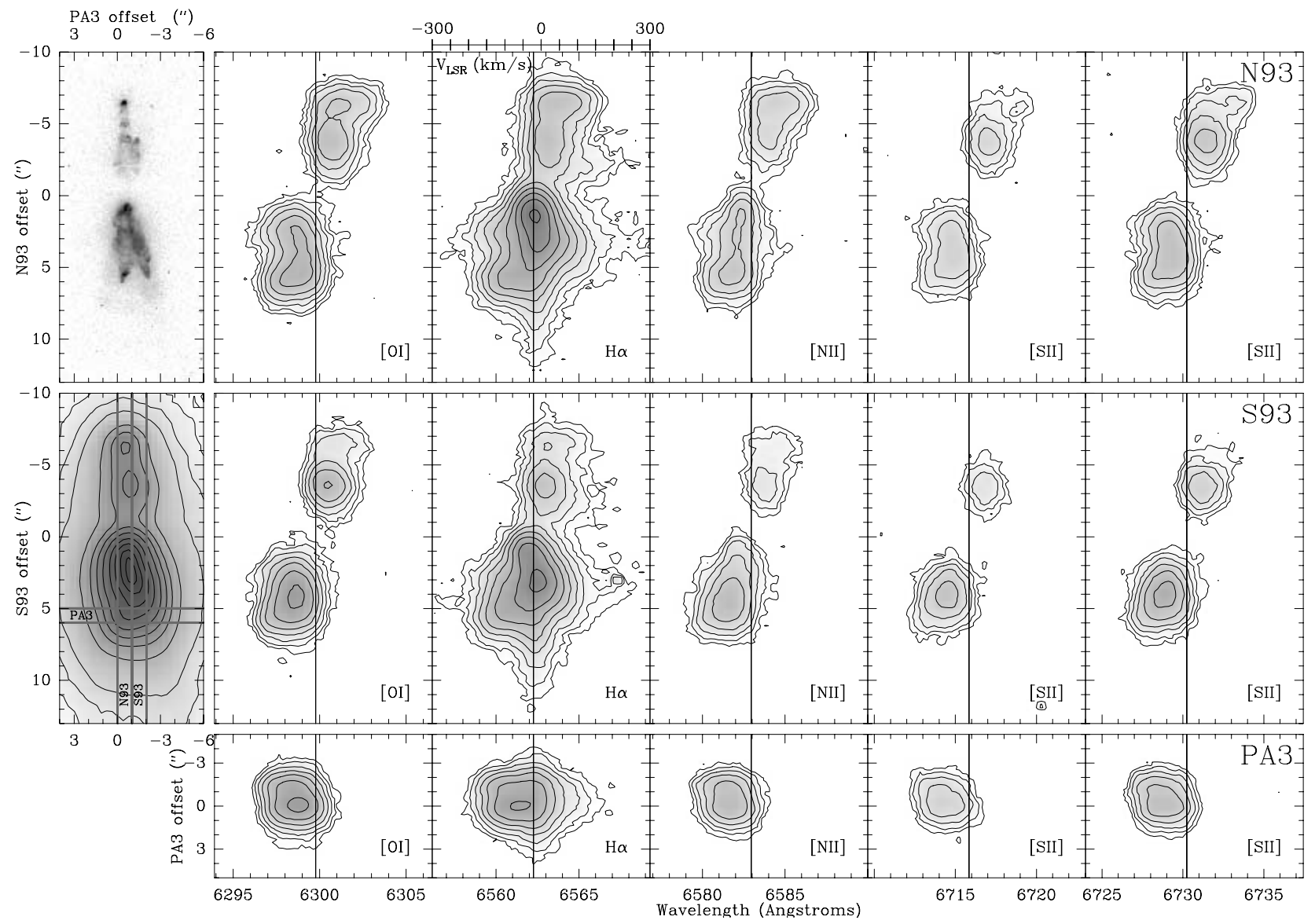

FIG. 2.- Long-slit spectra of selected emission lines in CRL 618 observed with grating R1200Y for slits N93 (top), S93 (middle), and PA3 (bottom). The HST and ground-based $\mathrm{H} \alpha$ images of the nebula are shown in the leftmost panels (top and bottom, respectively). Slits used for spectroscopy are superimposed on the ground-based image. Contours in the spectra are $3 \sigma \times 1.8^{(i-1)}$ from $i=1$ to 15 with $\sigma=10^{-16} \mathrm{ergs} \mathrm{s}^{-1} \mathrm{~cm}^{-2} \AA^{-1}$ arcsec ${ }^{-2}$ for N93 and $\sigma=1.4 \times 10^{-16}$ ergs s${ }^{-1} \mathrm{~cm}^{-2} \AA^{-1} \operatorname{arcsec}^{-2}$ for S93 and PA3. The LSR velocity scale (in $\mathrm{km} \mathrm{s}^{-1}$ ) is shown over the N93 $\mathrm{H} \alpha$ spectra. The systemic velocity (roughly $-21 \mathrm{~km} \mathrm{~s}{ }^{-1}$ ) is indicated by the vertical line.

pixels of $13.5 \mu \mathrm{m}$ lateral size. Only a clear and unvignetted region of $700 \times 2600$ pixels was used (the 2600 pixels were along the spectral axis). The CCD was mounted on the 500 $\mathrm{mm}$ camera, leading to a spatial scale of 0 .'19 pixel $^{-1}$. The $\mathrm{R} 1200 \mathrm{Y}$ and $\mathrm{R} 900 \mathrm{~V}$ gratings were employed providing spectra in the 6233-6806 and 4558-5331 $\AA$ wavelength ranges with dispersions of $16.4 \AA \mathrm{mm}^{-1}\left(0.22 \AA \mathrm{pixel}^{-1}\right)$ and $22.1 \AA \mathrm{mm}^{-1}\left(0.29 \AA\right.$ pixel $\left.^{-1}\right)$, respectively.

A total of three slit positions were observed at position angles of $93^{\circ}$ (along the nebular symmetry axis) and $3^{\circ}$ (through the west lobe of the nebula). The slit positions are shown in Figure 2 superimposed on the $\mathrm{H} \alpha$ ground-based image of CRL 618. At P.A. $=93^{\circ}$ two spectra were obtained: one with the slit placed on the northernmost pair of lobes (slit N93) and the other with the slit displaced $\sim 1^{\prime \prime}$ toward the south (partially covering the southernmost lobes; slit S93). At position N93, spectra with two gratings, R1200Y and R900V, were obtained with exposures times of 2000 and 2700 s, respectively. For the other two slit positions (S93 and P.A. $=3^{\circ}$ [slit PA3]) only the R1200Y was used, and exposure times were 2000 and 1300 s, respectively. The slit was $1^{\prime \prime}$ wide and long enough to cover the whole nebula (and a significant region of the sky).

The data were reduced following standard procedures for long-slit spectroscopy within the IRAF package, including bias subtraction, flat-fielding and illumination corrections, sky subtraction, and cosmic-ray rejection. We used $\mathrm{CuNe}$ and $\mathrm{CuAr}$ lamps to perform the wavelength calibration. The spectral resolution achieved (FWHM of the calibration lamp lines) is $\sim 50 \mathrm{~km} \mathrm{~s}^{-1}$ at $\mathrm{H} \alpha$ and $\sim 80 \mathrm{~km} \mathrm{~s}^{-1}$ at $\mathrm{H} \beta$. Flux calibration was done using sensitivity functions derived from two spectrophotometric standards, HR 1544 and HD 217086, observed at different air masses, and taking into account the atmospheric extinction curve at La Palma. The geometric distortion of the long-slit spectra $(\sim 0.06 \%)$ was corrected also using one of the flux calibration stars. The effective spatial resolution obtained ranges between $\sim 1^{\prime \prime}$ and 1 ". 6.

\section{MORPHOLOGY OF THE OPTICAL NEBULA}

In Figure 1 we show direct images of the PPN CRL 618 in the light of $(a)$ the $6616 \AA$ continuum and $\mathrm{H} \alpha$ line emission obtained (b) from the ground and (c) with HST. The $\mathrm{H} \alpha$ images show that the shocked, optical lobes of CRL 618 consist of several components with a very rich structure at a scale of $\sim 0$ ".2 (see Fig. 1c). The east and west lobes are both composed of several, collimated jetlike structures emanating from the center of the nebula. Bright, bow-shaped ripple-like features are frequent along the lobes.

In the $\mathrm{H} \alpha$ images of $\mathrm{CRL} 618$ we have labeled $\mathrm{A}^{\prime}$ the small, bright region of the east lobe closer to the nebular center (Fig. 1c). This region is very bright in the $\mathrm{H} \alpha$ image but very weak, e.g., in the WFPC2/HST [O I] $\lambda 6300$ and 
[S II] $\lambda \lambda 6716,6731$ images (see Trammell 2000; R. Sahai et al. 2002, in preparation). As we will see in the following sections, the spectrum of region $\mathrm{A}^{\prime}$ is in fact substantially different from that of the shock-excited lobes. This leads us to think that region $\mathrm{A}^{\prime}$ has a different nature, which we discuss in $\S 5.2$.

A weak $\mathrm{H} \alpha$ emission halo surrounds the lobes of CRL 618 (Figs. $1 b$ and $1 c$ ). In the HST $\mathrm{H} \alpha$ image the halo is observed very close to the boundaries of the emission lobes and is particularly intense and extended in the east lobe: note the clumps of diffuse emission ahead of this lobe (i.e., just beyond the tip of the lobe). The diffuse halo, like region $\mathrm{A}^{\prime}$, is absent in the [O I] and [S II] HST images (see references above). In our ground-based $\mathrm{H} \alpha$ image (with higher signalto-noise ratio than that obtained with the $H S T$ ) the size at a $3 \sigma$ level of the halo is $\sim 35^{\prime \prime} \times 22^{\prime \prime}$, with the major axis roughly oriented at P.A. $=3^{\circ}$ and centered at the position where the maximum $\mathrm{H} \alpha$ emission is observed.

The continuum image of CRL 618 traces the distribution of the dust particles in the nebula (which reflect the stellar light) as well as any field star. The central star of CRL 618 is not directly visible, which indicates that the stellar light is strongly obscured along the line of sight. The continuum brightness distribution is in general similar to that of $\mathrm{H} \alpha$, suggesting the presence of dust in the lobes.

\section{THE OPTICAL SPECTRUM OF CRL 618}

The optical spectrum of CRL 618 is composed of recombination and forbidden line emission superimposed on a faint, red continuum.

\subsection{Long-Slit Spectra of Emission Lines}

In Figure 2 we show long-slit spectra for the most intense lines in the $\sim 6230-6805 \AA$ range for slits N93, S93, and PA3. The weakest lines detected in this range, [S III] $\lambda 6312.1$ and He I $\lambda 6678$, for slit N93 are shown in Figure 3. These spectra have been smoothed with a flat-topped rectangular kernel of dimension $3 \times 3$ pixels; the resulting degradation

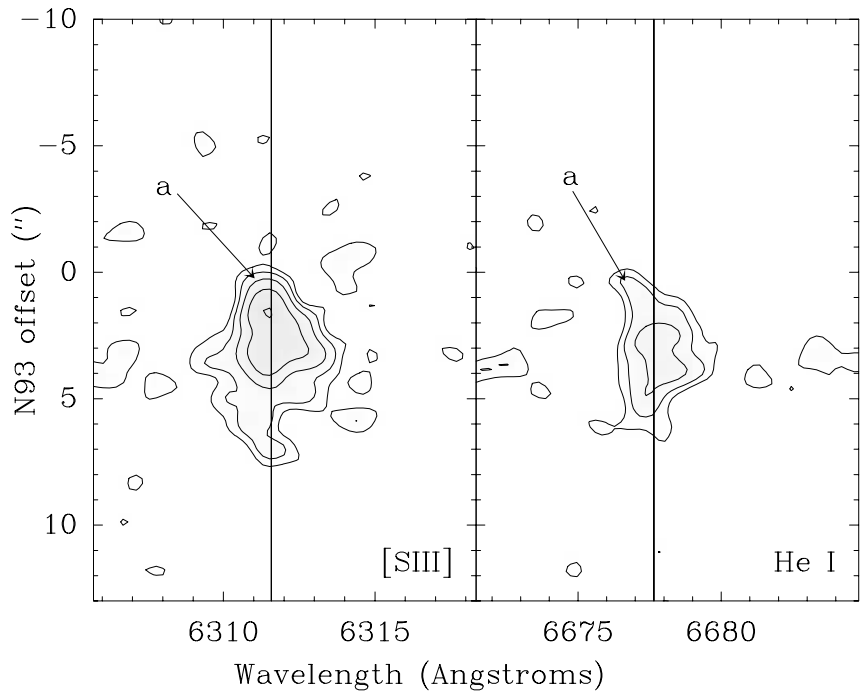

FIG. 3.-Smoothed long-slit spectra of [S III] $\lambda 6312.1$ and He I $\lambda 6678.2$ for slit N93. The total velocity range is $600 \mathrm{~km} \mathrm{~s}^{-1}$ as in Fig. 2. Contours are $2 \sigma \times 1.5^{(i-1)}$ from $i=1$ to 15 and with $\sigma=4 \times 10^{-17} \operatorname{ergs~s}^{-1} \mathrm{~cm}^{-2} \AA^{-1}$ $\operatorname{arcsec}^{-2}$. The vertical line is placed at LSR $V_{\text {sys }}$ (roughly $-21 \mathrm{~km} \mathrm{~s}^{-1}$ ). Feature "a" is indicated (see also Fig. 4). in the spatial and spectral resolution is less than $\sim 4 \%$ from the nominal value (see $\S 2.2$ ) since the smoothing window was smaller than the local seeing and FWHM of the calibration lines. The origin of the spatial scale in the spectra (and in the direct images of the nebula, Figs. 1 and 2) coincides with the point of maximum extinction (measured as the highest $\mathrm{H} \alpha / \mathrm{H} \beta$ line ratio; $\S 8)$. We will refer to this point also as to nebular center. The LSR systemic velocity of the source, $V_{\text {sys }}=-21.5 \pm 0.5 \mathrm{~km} \mathrm{~s}^{-1}$ (derived from molecular line emission observations; e.g., Cernicharo et al. 1989), is indicated by a vertical line on each spectrum.

We find remarkable differences between the profile of the $\mathrm{H} \alpha$ line (and also $\mathrm{H} \beta$; see below) and those of most forbidden lines for each slit position. These differences are specially noticeable in the bright east lobe. A number of spectral features $^{2}$ (labeled A, B, and C in Fig. 4) are seen to be superimposed on an underlying $\mathrm{H} \alpha$ profile very similar

2 "Feature" refers to a component in our two-dimensional spectra.
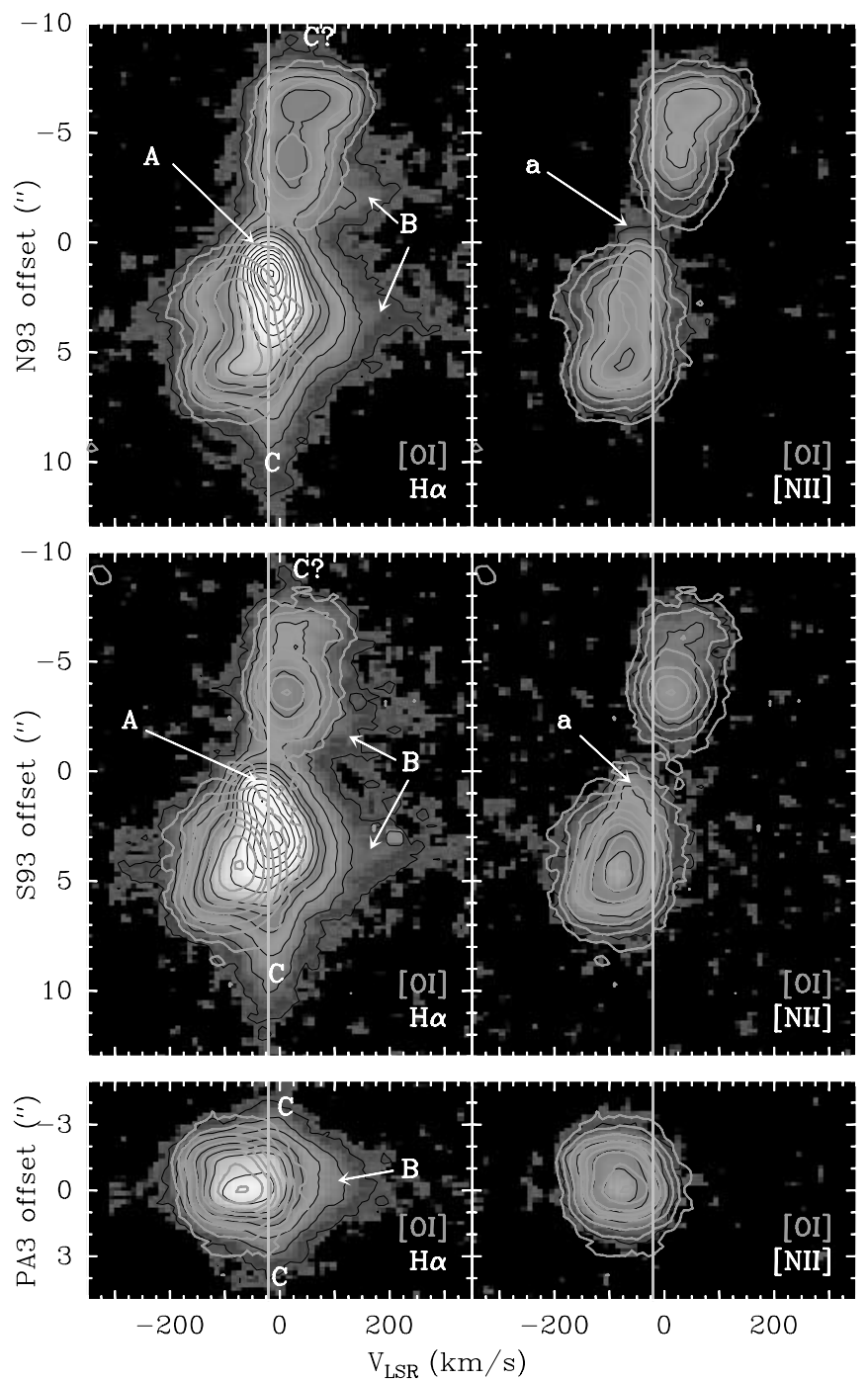

FIG. 4.-Long-slit spectra of $\mathrm{H} \alpha$, [N $\mathrm{II}] \lambda 6584$ (gray scale and black contours), and [O I] $\lambda 6300$ emission (light gray contours) for slits N93 (top), S93 (middle), and PA3 (bottom). The intensity levels represented are the same for all transitions. Note that except for the presence of features $\mathrm{A}, \mathrm{B}$, and $\mathrm{C}$ in the $\mathrm{H} \alpha$ spectrum, the profiles and fluxes of $\mathrm{H} \alpha$ and [O I] are comparable. In the [N II] profile, feature "a" is the counterpart to feature A. [See the electronic edition of the Journal for a color version of this figure.] 
to that of [O I] $\lambda 6300$ (and most forbidden lines; CS82 noted also the presence of two different components in the $\mathrm{H} \alpha$ profile; see $\S 5.1$ ).

Feature $\mathrm{A}$ is the intense $\mathrm{H} \alpha$ emission component observed in the innermost region (closest to the central star) of the east lobe. The maximum emission from this feature occurs at offset $\sim 1 " 4$. For slit N93 such maximum is slightly redshifted $\left(\sim 3.5 \mathrm{~km} \mathrm{~s}^{-1}\right)$ with respect to $V_{\text {sys }}$, whereas for $\mathrm{S} 93$ feature $\mathrm{A}$ is blueshifted by $\sim 10 \mathrm{~km} \mathrm{~s}^{-1}$. From comparison with the $\mathrm{H} \alpha$ images of CRL 618 we determine that feature $\mathrm{A}$ originates in the inner region $\mathrm{A}^{\prime}$ (Fig. 1). There is also some blueshifted [N II] $\lambda \lambda 6548,6584$, [S III] $\lambda 6312$, and He I $\lambda 6678$ emission arising in this region, which we refer to as emission feature " a " by analogy to feature $\mathrm{A}$ in the $\mathrm{H} \alpha$ profile (Figs. 3 and 4). Feature B is the broad, redshifted wing around an offset of roughly -2.5 and 3".5 in our N93 and S93 spectra. This feature is prominent in regions where the scattered continuum reaches maximum intensity (see Fig. 5 and the continuum image in Fig. 1). Feature B is also present in the $\mathrm{PA} 3 \mathrm{H} \alpha$ spectra. Feature $\mathrm{C}$ is the slightly redshifted emission component observed close to the systemic velocity from offsets $6^{\prime \prime}$ to $12^{\prime \prime}$ in our N93 and S93 spectra. We note that feature $\mathrm{C}$ extends beyond the region where forbidden line emission is observed (i.e., beyond the shocked lobes). From comparison with the $\mathrm{H} \alpha$ direct images of the nebula we conclude that feature $\mathrm{C}$ originates in the weak "halo" that surrounds the bright lobes (Fig. 1). This halo is visible toward the east up to offsets $20^{\prime \prime}$ in our deep, $\mathrm{H} \alpha$ ground-based image. Feature $\mathrm{C}$ is also present along slit PA3, which crosses the lobes and the halo at the outermost parts of the east lobe. We have labeled " $\mathrm{C}$ ?" the weak, red- shifted emission beyond the west lobe in slits N93 and S93 (Fig. 4). This tentative feature (also marginally present in the $\mathrm{H} \beta$ profile) has a redshift slightly larger than that of feature C.

For slit S93, the maxima in the $\mathrm{H} \alpha$ and forbidden (e.g., [O I] line emission are observed at different spatial and spectral positions: the $\mathrm{H} \alpha$ emission peak is redshifted and located at offset $\sim 3$." 5 , whereas the maximum emission from most forbidden lines is clearly blueshifted and located at offset $\sim 4$ ".2. The reason for this difference is that the $\mathrm{H} \alpha$ emission peak is most likely the result of the superposition of features C, B, and maybe A on top of the lobe emission and not only emission produced locally in the east lobe.

In Figure 5 we present the long-slit spectra obtained along the nebular axis (N93) in the wavelength range 4558$5331 \AA$. The $\mathrm{H} \beta$ profile is similar to that of $\mathrm{H} \alpha$, except that feature $\mathrm{A}$ is less prominent for the former, very likely as a result of a larger extinction toward the nebular center. Features $\mathrm{B}$ and $\mathrm{C}$ are clearly detected in the $\mathrm{H} \beta$ profile up to (projected) velocities of $\sim 290 \mathrm{~km} \mathrm{~s}^{-1}$ (LSR) and around $V_{\text {sys }}$, respectively.

The profile of [O III] $\lambda 5007$ is similar to that of $\mathrm{H} \beta$ : note the maximum emission slightly redshifted at the position where the continuum is detected in our spectra (coincident with feature $\mathrm{B}$ in $\mathrm{H} \alpha$ ) and the weak emission closer to the nebular center (coincident with feature A). The profile of the $[\mathrm{N}$ I] doublet (partially resolved) is different from $\mathrm{H} \beta$ and [O III] but similar to most forbidden lines in the red.

We have detected a number of weak emission lines in this wavelength range, most of them from $\mathrm{Fe}$ ions. The [Fe III] lines, similarly to the [S III] $\lambda 6312$ line, are observed in the
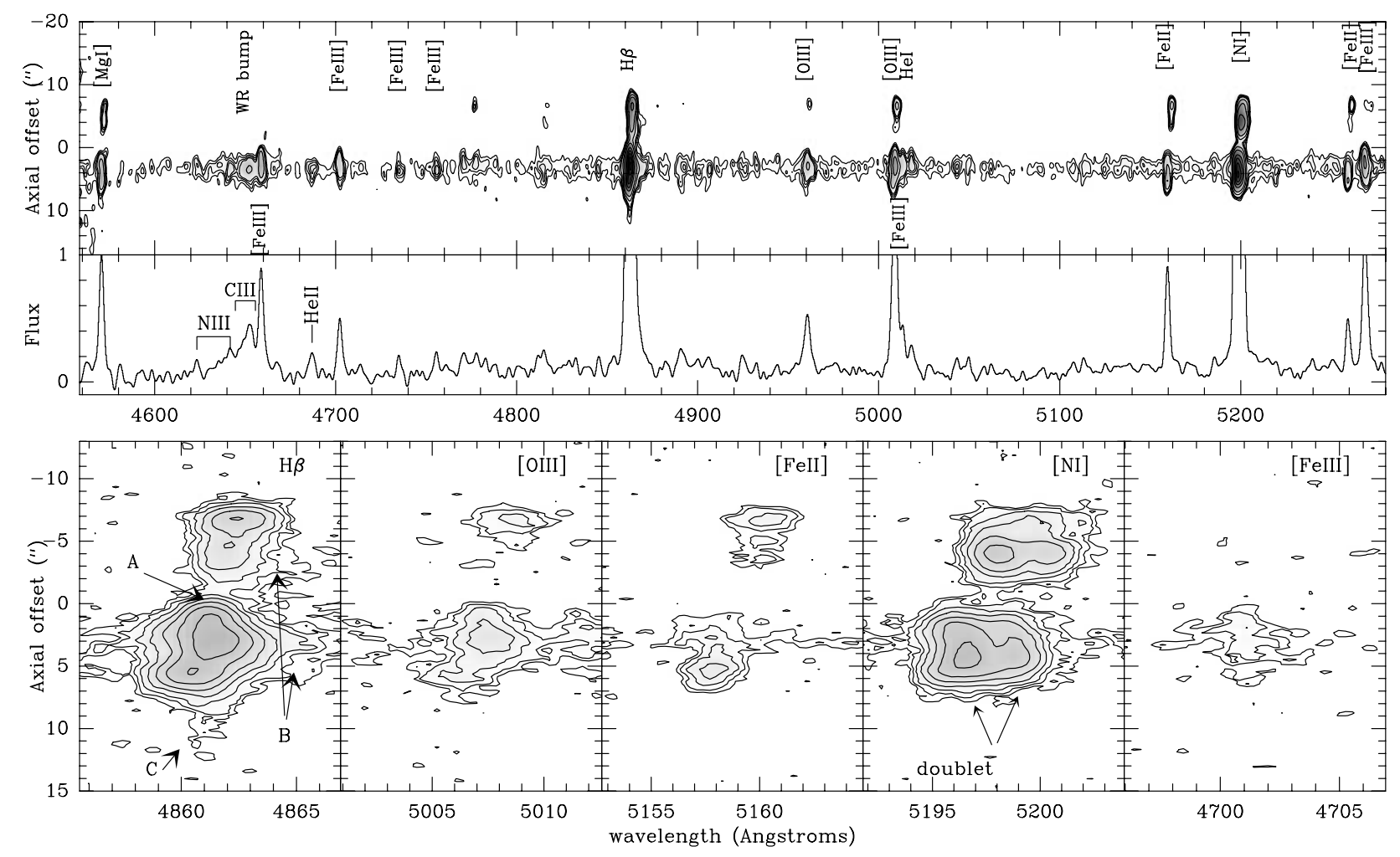

FIG. 5.- Long-slit spectra in the blue (grating R900V) obtained along the axis of CRL 618 (position N93 in Fig. 2). In the top panel, we plot the total spectrum with degraded spatial and spectral resolutions by a factor of $70 \%$ and $50 \%$, respectively, with respect to the nominal value in order to show the continuum and weakest emission lines. In the bottom panel, long-slit spectra for selected lines are shown. As in Fig. 2, these spectra have been smoothed within a $3 \times 3$ pixel box with no significant degradation (less than $4 \%$ ) of the nominal spatial and spectral resolution (see $\S 2.2$ ). 
region where the scattered continuum reaches its maximum intensity and in the inner region where feature A arises, with no trace of emission from the outer parts of the shocked lobes. The $[\mathrm{Fe}$ II] lines are remarkably intense at the tips of the lobes and show no sign of $\mathrm{H} \alpha$ analog features; i.e., they show no emission from region $\mathrm{A}^{\prime}$ or the region of maximal continuum emission.

At $4650 \AA$, we also detect a faint and broad emission feature, which is only visible in the region where the continuum is observed for the east lobe. We identify this feature with the Wolf-Rayet (W-R) bump at $4650 \mathrm{~A}$ (see below).

\subsection{Line Identification and Flux Measurements}

We list in Table 1 all the lines that we have detected in the optical spectra of CRL 618 together with their air wavelengths and fluxes (undereddened). The fluxes have been estimated from slit N93 (for other slits only the lines in the red wavelength range were observed), integrating the emission of the pixels above the $3 \sigma$ level along the slit and in the spectral direction.

Most of the lines in Table 1 were already detected and identified in this source from previous optical spectroscopy (see references in $\S 1$ ); however, we find some new features in this work. The most relevant of the new identifications is the W-R bump at $\sim 4650 \AA$ (Fig. 5). This bump is a blend of high-excitation lines such as $\mathrm{N}$ III $\lambda \lambda 4634-4641$ and $\mathrm{C}$ III $\lambda \lambda 4647-4651$ (e.g., Tylenda, Acker, \& Stenholm 1993). The $\mathrm{C}$ IV $\lambda 4658.3$ line, usually considered as a part of the W-R bump, is listed separately in Table 1 together with the

TABLE 1

ObSeRVEd Line FluXes For CRL 618

\begin{tabular}{|c|c|c|}
\hline \multirow[b]{2}{*}{ LiNe } & \multicolumn{2}{|c|}{$\begin{array}{c}\text { FLUX } \\
\left(\times 10^{-15} \mathrm{ergs} \mathrm{s}^{-1} \mathrm{~cm}^{-2}\right)\end{array}$} \\
\hline & West & East \\
\hline 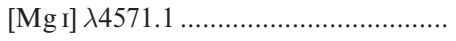 & $1.2(0.3)$ & $3.5(0.9)$ \\
\hline N v $\lambda 4620, N_{\text {III }} \lambda \lambda 4634,4641$ & & \\
\hline C III $\lambda \lambda 4647,4650$ (W-R bump) ....... & $\ldots$ & $6(2)$ \\
\hline$[\mathrm{Fe}$ III] $\lambda 4658.05, \mathrm{C}$ IV $\lambda 4658.30 \ldots \ldots \ldots . .$. & $\ldots$ & $3.7(0.8)$ \\
\hline Не II $\lambda 4685.55 \ldots \ldots \ldots$ & $\ldots$ & $0.8(0.2)$ \\
\hline 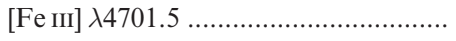 & $\ldots$ & $1.7(0.4)$ \\
\hline 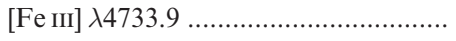 & $\ldots$ & $0.5(0.4)$ \\
\hline 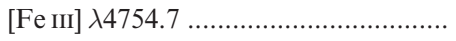 & $\ldots$ & $0.5(0.4)$ \\
\hline $\mathrm{H} \beta \lambda 4861.3 \ldots \ldots$. & $11(1)$ & $46(4)$ \\
\hline$[\mathrm{O}$ III $] \lambda 4958.9 .$. & $0.4(0.2)$ & $2(1)$ \\
\hline 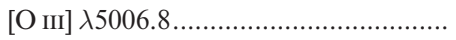 & $1.3(0.2)$ & $6(1)$ \\
\hline$[\mathrm{Fe}$ III] $\lambda 5011.3 \ldots \ldots \ldots \ldots \ldots \ldots \ldots \ldots \ldots$ & $\ldots$ & $1(0.6)$ \\
\hline Не г $\lambda 5015.7 \ldots \ldots \ldots$ & $\ldots$ & $0.6(0.7)$ \\
\hline 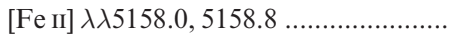 & $1.5(0.2)$ & $2.5(0.7)$ \\
\hline 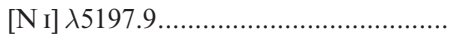 & $9.5(0.3)$ & $23(1)$ \\
\hline 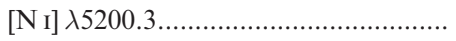 & $5.8(0.3)$ & $11.0(0.7)$ \\
\hline$[\mathrm{Fe}$ II $] \lambda 5261.6 \ldots \ldots$. & $0.9(0.3)$ & $1.2(0.6)$ \\
\hline 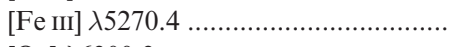 & $\ldots$ & $4.3(0.6)$ \\
\hline$[\mathrm{O}$ I] $\lambda 6300.3 \ldots \ldots \ldots$ & $55(3)$ & $94(4)$ \\
\hline 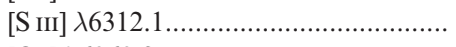 & $\ldots$ & $2.5(0.6)$ \\
\hline 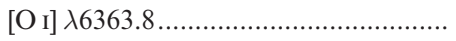 & $18(2)$ & $33(3)$ \\
\hline 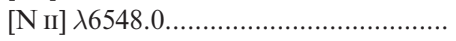 & $10(2)$ & $16(2)$ \\
\hline 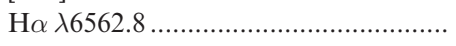 & $83(4)$ & $390(8)$ \\
\hline 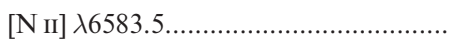 & $33(3)$ & $56(3)$ \\
\hline 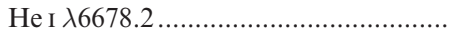 & $\ldots$ & $1.8(0.8)$ \\
\hline 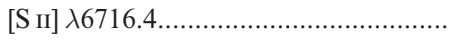 & $18(2)$ & $34(3)$ \\
\hline 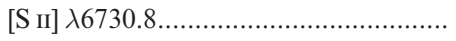 & $35(2)$ & $60(3)$ \\
\hline
\end{tabular}

NoTE.-Errors are given in parentheses. blended [Fe III] $\lambda 4658.1$ line because it is spectrally resolved from the rest of the bump in our spectra. The W-R bump ${ }^{3}$ is detected in the east lobe, only in the region where the scattered continuum (and feature B) is observed. The onedimensional blue spectrum for this region is shown in Figure 5, where the presence of He II $\lambda 4685.55$ (also reported for the first time in this work) and other high-excitation (Fe?) lines can be noticed.

Emission around $5007 \AA$ was detected previously with a low signal-to-noise ratio and attributed to a blend of [Fe II] and [O III] lines (Schmidt \& Cohen 1981; Kelly et al. 1992). We have also detected the [O III] $\lambda 4959$ line in our deep spectra with a total flux $\sim \frac{1}{3}$ of the [O III] $\lambda 5007$ line (Table 1) as theoretically expected regardless of the nebular conditions. Accordingly, the flux measured at $5007 \AA$ must be mostly due to the $[\mathrm{O}$ III] $\lambda 5007$ line.

We have compared the absolute fluxes measured in the present work with the fluxes measured by Kelly et al. (1992). For most of the lines we find good agreement, our fluxes being on average $\sim 2.2$ and $\sim 1.6$ (for the east and west lobes, respectively) times smaller than those measured by Kelly et al. (1992), as expected from the different slit widths used in both observations (they use a 2 ". 5 wide slit, covering the nebula almost totally in the equatorial direction and totally along the axis). There is however a remarkable difference in our fluxes for the [O III] $\lambda \lambda 4959,5007$ and [O I] $\lambda \lambda 6300,6363$ lines and those measured by Kelly et al. (1992). For the [O III] transitions, these authors found a total flux for the east (west) lobe a factor of $\sim 0.8$ (1) smaller than ours, without correcting the different slit width. Considering that our slit is a factor of $\sim 2.5$ smaller than theirs and the size of the nebula (2".5-3" in the direction perpendicular to the slit), we must conclude that the intensity of the [O III] lines is now a factor of 2-3 larger than 9 years ago. Trammell et al. (1993) also found upper limits for the flux of the [O III] lines that are also much smaller than our measurements in spite of their broader slit $\left(2^{\prime \prime}\right)$. We also derive an $[\mathrm{O} \mathrm{III}] / \mathrm{H} \beta$ line ratio (less dependent on the slit width) a factor of $\sim 2$ larger than that obtained by Kelly et al. (1992) in agreement with a recent increase of the [O $\mathrm{III}]$ intensity.

For the [O I] transitions, Kelly et al. (1992) measure fluxes a factor of $\sim 3.5$ and $\sim 2$ (for the east and west lobe, respectively) larger than ours. Correcting by the different slit width, the intensity of the [O I] lines has decreased by a factor of $\sim 0.6$ and 0.8 since 1990 . The values of the $[\mathrm{O}$ I] $/ \mathrm{H} \alpha$ line ratio measured in previous works, summarized in Table 2 and Figure 6, confirm the progressive weakening with time of the $[\mathrm{O} \mathrm{I}]$ transitions.

\subsection{Continuum}

A relatively weak continuum is clearly visible in the blue (R900V) long-slit spectra along the nebular axis around offset $3^{\prime \prime}$ (on the bright east lobe; see Fig. 5, top panel). This position corresponds to the relative maximum in the continuum brightness distribution seen in the direct image (Fig. 1).

\footnotetext{
${ }^{3}$ The $\mathrm{C}$ III/C IV lines in the W-R bump are observed in the majority of [WC]-type central stars of PNs (Leuenhagen, Hamann, \& Jeffery 1996), but also in some normal PNs (i.e., with no [WC]-type central stars; Aller \& Keyes 1987). In the latter, where $\mathrm{N}$ III lines are also found, these high-excitation transitions are the result of nebular emission. Therefore, the presence of the W-R bump in the spectrum of CRL 618, while potentially interesting and deserving further investigation, is not sufficient to conclude that it has a [WC]-type central star.
} 
TABLE 2

[O I] $\lambda 6300 / \mathrm{H} \alpha$ LINE RATIOS FOR CRL 618

\begin{tabular}{|c|c|c|c|c|}
\hline \multirow[b]{2}{*}{ OBSERVATION Date } & \multirow{2}{*}{$\begin{array}{c}\text { JD } \\
\text { (days) }\end{array}$} & \multicolumn{2}{|c|}{$\left[\mathrm{O}_{\mathrm{I}}\right] / \mathrm{H} \alpha$} & \multirow[b]{2}{*}{ REFERENCE } \\
\hline & & East & West & \\
\hline 1973 Oct-1974 Feb....... & $2,442,032$ & $1.2^{\mathrm{a}}$ & $1.2^{\mathrm{a}}$ & 1 \\
\hline 1979 Dec .................... & $2,444,223$ & 0.9 & 1.3 & 2 \\
\hline 1989 Feb-1990 Oct........ & $2,447,846$ & 0.4 & 0.8 & 3 \\
\hline 1990 Oct-Nov ................ & $2,448,197$ & 0.5 & 0.3 & 4 \\
\hline 1994 Oct........................... & $2,449,641$ & 0.4 & 1.1 & 5 \\
\hline 2000 Nov ….................... & $2,451,864$ & 0.2 & 0.7 & 6 \\
\hline
\end{tabular}

${ }^{\text {a }}$ Ratio obtained for the whole nebula.

ReFERENCES.- (1) Westbrook et al. 1975. (2) Schmidt \& Cohen 1981. (3) Kelly et al. 1992. (4) Trammell et al. 1993. (5) Baessgen et al. 1997. (6) This paper.

We also detect continuum emission at the same position in the red spectra (R1200Y) for slits N93 and S93, as well as at the spatial origin for slit PA3, but with smaller signal-tonoise ratio. The red continuum is only visible in substantially smoothed spectra (not shown here). The average surface brightness of the red (blue) continuum, measured from our long-slit spectra in a $1^{\prime \prime} \times 1^{\prime \prime}$ region around offset $3^{\prime \prime}$, is $\sim 8 \times 10^{-17}\left(\sim 5 \times 10^{-17}\right) \operatorname{ergs~s}^{-1} \mathrm{~cm}^{-2} \AA^{-1} \operatorname{arcsec}^{-2}$. The continuum brightness has been measured in a spectral window of $80 \AA$ in the vicinity of $\mathrm{H} \alpha$ and $\mathrm{H} \beta$, respectively. The intensity and spatial distribution of the continuum obtained from our long-slit spectra are consistent with the continuum image (Fig. 1).

Our measurements of the red continuum flux are in reasonable agreement with previous estimates by Kelly et al. (1992). Nevertheless, the continuum at blue wavelengths in our spectra is only a factor of $\sim 1 / 1.6$ weaker than the red, whereas from the data given by the previous authors we measure a blue-to-red continuum ratio of $\sim 1 / 2.4$. Note that although the absolute flux calibration can be uncertain up to a factor of $\sim 50 \%$, the relative flux value is more accurate. This difference is consistent with a blue brightening of a factor of $\sim 1.5(\sim 0.44 \mathrm{mag})$ in the last 10 years (assuming that the red continuum flux has not varied). Gottlieb \& Liller (1976) reported an increase of $\sim 2 \mathrm{mag}$ in $B$ band, from

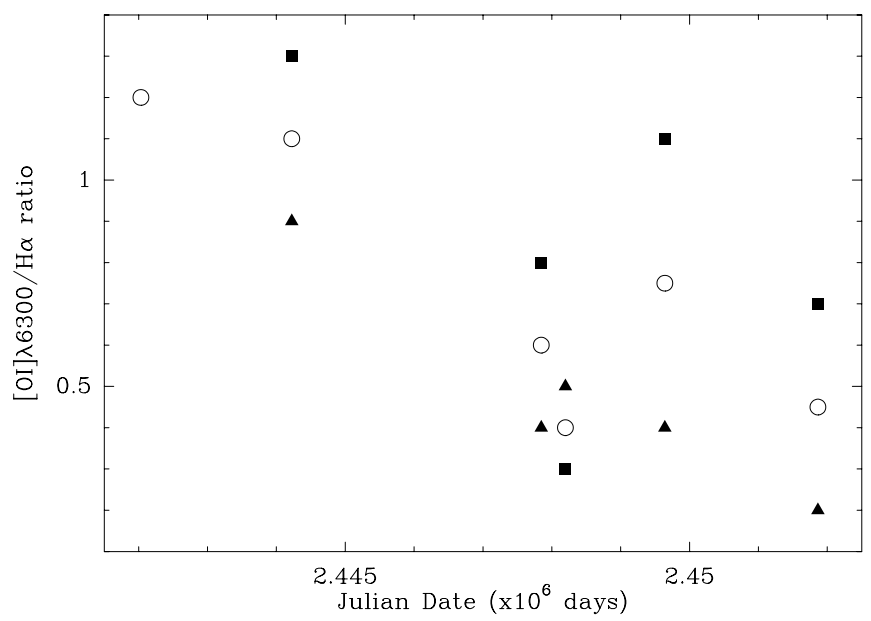

FIG. 6.- [O I] $\lambda 6300 / \mathrm{H} \alpha$ line ratio measured in CRL 618 by different authors (see Table 2). Filled triangles (squares) are used for the east (west) lobe, and open circles for average values in the whole nebula. measurements between 1922 and 1975. These authors

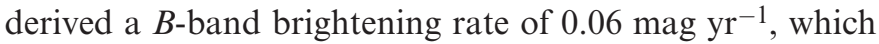
is roughly consistent with our results. These authors attributed the increase of the blue continuum flux to the expansion of the CSE of CRL 618 and the subsequent decrease of the extinction.

\section{SPECTRAL COMPONENTS AS TRACERS OF DIFFERENT NEBULAR REGIONS}

\subsection{Scattered and Local Emission Components}

We find remarkable differences between the long-slit profiles of the recombination and forbidden emission lines in CRL 618. Figures 2 and 4 clearly show the presence of three "extra" emission features (A, B, and C) in the $\mathrm{H} \alpha$ spectrum compared to the profile of most forbidden lines. Some of these features are also present in certain forbidden lines $(\S 4.1)$. Here we analyze the origin of these features.

Feature $A$, the intense $\mathrm{H} \alpha$ emission observed close to the nebular center in slits $\mathrm{N} 93$ and S93, arises in the inner region $\mathrm{A}^{\prime}$ (Fig. 1). This region is very bright in the HST $\mathrm{H} \alpha$ image but much weaker in the light of [O I] and [S II] (Trammell 2000). This behavior is consistent with the absence of feature $\mathrm{A}$ in our spectra for these forbidden transitions. For slit $\mathrm{S} 93$, feature A is blueshifted with respect to $V_{\text {sys }}$ by $\sim 10 \mathrm{~km}$ $\mathrm{s}^{-1}$. The counterpart to this feature observed in other recombination and forbidden lines is also blueshifted: for [N II] $\lambda 6584$, feature " a" is blueshifted by $\sim 25$ and $33 \mathrm{~km}$ $\mathrm{s}^{-1}$ at position $\mathrm{N} 93$ and $\mathrm{S} 93$, respectively; for $\mathrm{H} \beta$, [O III] $\lambda 5007$, and [S III] $\lambda 6312$, observed only at position N93, the blueshift is $\sim 8-12 \mathrm{~km} \mathrm{~s}^{-1}$. The measured Doppler blueshift suggests that feature $A$ is locally produced within the east lobe, which is moving toward us. For slit N93, feature A lies at $V_{\text {sys }}$, showing no apparent shift toward the blue. We believe that in this case the spectral position of the $\mathrm{H} \alpha$ feature A could be altered (slightly redshifted) because of the presence of feature B at almost the same spatial position, which is relatively intense and is clearly redshifted in the east lobe. The blueshift of feature A could also be intrinsically smaller for position N93 than for S93, as suggested by the smaller blue velocities of its counterpart (feature "a") in the other lines.

Features $B$ and $C$ are most likely the scattered component of the emission in the Balmer lines for this source $(\S 1)$. Previous spectropolarimetric observations indicate that $\sim 40 \%$ of the total $\mathrm{H} \alpha$ flux is polarized (with a line polarization of about $15 \%$; Trammell et al. 1993); i.e., it is light scattered by nebular dust. The polarization of the forbidden lines is, however, much lower, and this would explain the absence of these features in these cases. Features B and C are redshifted, although they originate in the approaching lobe (note that the forbidden line emission from the east lobe is blueshifted), which clearly points to their scattered nature: the light scattered by dust moving away from the central source should be redshifted for both the approaching and receding lobes (see, e.g., Schwarz et al. 1997). CS82 also found some redshifted $\mathrm{H} \alpha$ emission (probably our feature B) arising in the approaching bright lobe, which they attribute to scattered emission following the same argument.

The previous interpretation is consistent with the similar brightness distribution of these features and the continuum (Figs. 1 and 2), as expected if they share the same scattered nature. Feature B is clearly most prominent in the region of 
maximum continuum emission for all the slit positions. Moreover, features $\mathrm{B}$ and $\mathrm{C}$ are much more intense for the east lobe than for the west one, where the scattered continuum is much weaker and the fraction of polarized light smaller ( 0.5 vs. 0.2 for the east and west lobes, respectively; Schmidt \& Cohen 1981).

The different profiles and spatial distributions of features $\mathrm{B}$ and $\mathrm{C}$ in our long-slit spectra suggest that they are produced by dust in different nebular regions. The low redshift of feature $\mathrm{C}\left(\sim 6-8 \mathrm{~km} \mathrm{~s}^{-1}\right)$ and its location (beyond the forbidden line-emitting east lobe, in the weak $\mathrm{H} \alpha$ halo) point to dust not located inside the lobe but beyond it, in the unshocked, slowly expanding AGB CSE. Since the AGB CSE surrounds the lobes, we can expect feature $C$ to be present (with more or less intensity) all along the slits. This is consistent with the presence of the diffuse halo surrounding the lobes in the $\mathrm{H} \alpha$ images and not only in front of them (see below). The tentative feature "C?," observed at the tip of the west lobe, could be the counterpart to feature $\mathrm{C}$ in the receding lobe.

The much larger redshift of feature B (up to LSR velocities of $\sim 240 \mathrm{~km} \mathrm{~s}^{-1}$ ) and its location (within the lobes) are more consistent with dust inside the shocked lobes that is flowing outward rapidly (presumably at the same speed as the gas). We identify this feature with the redshifted $\mathrm{H} \alpha$ emission detected by CS82 in the east lobe, which they also attribute to scattered emission produced by fast outflowing dust. At the position where feature B is observed, some contribution by feature $\mathrm{C}$ is also expected (see above); however, the spectral resolution achieved in these observations is not sufficient to separate both features.

The line emission that is being reflected by the dust, visible as the halo (in the direct images) and as features B and C (in our long-slit spectra), is unlikely to originate in the shocked lobes themselves. In fact, neither the halo nor features $\mathrm{B}$ and $\mathrm{C}$ are detected in most forbidden lines and, in particular, in the $\left[\mathrm{O}_{\mathrm{I}}\right]$ line. However, the $\left[\mathrm{O}_{\mathrm{I}}\right]$ emission and $\mathrm{H} \alpha$ emission locally produced in the east lobe have comparable intensities (Fig. 4 and HST images in Trammell 2000), which should result in almost equally bright scattered components for both lines if the reflected photons were originally produced in the lobes. Features B and C must then result from the reflection of light produced in the nebular center (in the central $\mathrm{H}$ II region and/or in region $\mathrm{A}^{\prime}$ ), where the emission of most forbidden lines observed by us is intrinsically much less intense.

\subsection{The Different Nebular Regions}

The presence or absence of the different spectral features and, in general, the profiles of the different lines are diagnostic of the physical properties (such as temperature, ionization, and electron density) of different nebular regions in CRL 618, from the highly obscured $\mathrm{H}$ II region to the shocked lobes.

\subsubsection{The Inner $\mathrm{H}$ iा Region}

This region is indirectly probed by lines with B- or C-like features (scattered light), namely, $\mathrm{H} \alpha, \mathrm{H} \beta,[\mathrm{O}$ III], He I, He II, [Fe III], [S III], and the W-R bump. The spectrum of the central $\mathrm{H}$ II region is thus most easily seen in the region where the scattered continuum is observed, at offset $\sim 3^{\prime \prime}$ in the east lobe. The central H II region (previously studied through its radio continuum emission; see $\S 1$ ) is character- ized by high densities $\left(\gtrsim 10^{6} \mathrm{~cm}^{-3}\right)$ and high temperatures $(13,000-15,000 \mathrm{~K})$ that provide the required conditions to produce most of the (high-excitation) lines above (e.g., the ionization potentials of the [Fe III], [S III], He II, and W-R bump are $16.16,23.33,24.59$, and $\gtrsim 30 \mathrm{eV}$, respectively). The large critical densities for the observed [Fe III] and [S III] transitions, $n_{c} \sim 10^{8}-10^{9} \mathrm{~cm}^{-3}$, explain why these forbidden lines are not de-excited by collisions in the central region of CRL 618. We note also that the relatively large critical density of the [O III] $\lambda 5007$ transition, a few times $10^{5} \mathrm{~cm}^{-3}$, would explain the weak [O III] $\lambda 5007$ emission from the inner $\mathrm{H}$ II region, which is indirectly observed at the position of maximal continuum and feature B. Unfortunately, spectropolarimetry of the lines listed above presumably arising from the $\mathrm{H}$ II region has not been obtained previously, so we do not know their polarization degree (nor the fraction of scattered flux) and cannot test the previous scenario. In any case it seems that these high-excitation lines are unlikely to be produced in the shocked lobes, where the spectrum is dominated by low-excitation lines.

\subsubsection{Region $A^{\prime}$}

This region is also very likely a relatively dense region, considering that only forbidden lines with high critical densities $\left(n_{c} \gtrsim\right.$ few times $\left.10^{4} \mathrm{~cm}^{-3}\right)$ are observed therein, namely, [N II], [S III], [O III], and [Fe III]. The spectrum of this region (the innermost part of the east lobe, where features A and "a" are observed) is remarkably different from that of the shocked lobes but very similar to that of the central H II region. In fact, most lines are common to both regions except for the absence of the W-R bump and the He II line (which require higher excitation conditions) in region $\mathrm{A}^{\prime}$ and the undetected [N II] emission (with a low critical density, $n_{c} \sim 2 \times 10^{4} \mathrm{~cm}^{-3}$ ) from the denser $\mathrm{H}$ II region. This fact suggests that region $\mathrm{A}^{\prime}$ is more likely to be ionized by the UV stellar radiation rather than by shocks. In this sense, region $\mathrm{A}^{\prime}$ represents the outermost (less dense) parts of the $\mathrm{H}$ II region surrounding the star. The observed relative line intensities are not consistent with any of the different shock excitation models by Hartigan, Raymond, \& Hartmann (1987, hereafter HRH87).

The very weak emission of the $[\mathrm{O} \mathrm{I}]$ lines from region $\mathrm{A}^{\prime}$ ([O I $] / \mathrm{H} \alpha<0.05)$ implies that this region is fully ionized (e.g., Hartigan, Morse, \& Raymond 1994). The observed $\left[\begin{array}{ll}\mathrm{O} & \mathrm{I}\end{array}\right]$ transitions have relatively high critical densities $\left(n_{c} \sim 2 \times 10^{6} \mathrm{~cm}^{-3}\right)$; therefore, significant collisional deexcitation of the involved levels is not expected. Thus, the weakness of the $\left[\mathrm{O}_{\mathrm{I}}\right]$ transitions is because most oxygen in region $\mathrm{A}^{\prime}$ is ionized. Considering the similar ionization potentials of oxygen and hydrogen, we conclude that the ionization fraction $\left(X=n_{e} / n_{\text {total }}\right)$ of region $\mathrm{A}^{\prime}$, like that of the inner $\mathrm{H}$ II region (where $[\mathrm{O} \mathrm{I}]$ is not detected), is $\sim 1$.

\subsubsection{The Shocked Lobes}

This region is probed by lines without any additional features $(\mathrm{A}, \mathrm{B}$, or $\mathrm{C})$. The most intense of these lines ([O I], [S II], and [N I]) are known to originate entirely from the gas in the lobes since for these lines, no polarization has been measured (Trammell et al. 1993) and no redshifted components are found in the approaching lobe. Although no polarimetry of the rest of the lines within this class has been made, we conclude that they must have a similar origin given their similar profiles. 
The relative intensities of the lines observed at the lobes (dereddened using the average extinction for each lobe; see $\S 8$ below) are consistent with shock-excited emission. In particular, the flux ratios observed by us lie in between the predicted values for bow shock excitation models 8 and 9 by HRH87 with shock velocities between 50 and $100 \mathrm{~km} \mathrm{~s}^{-1}$ and preshock density $300 \mathrm{~cm}^{-3}$. This range of velocities for the shocks is mainly constrained by the detected, although weak, [O III] $\lambda 5007$ emission and implies shock speeds slightly larger than (but still comparable to) previous estimates following similar procedures $\left(V_{\text {shock }}=20-80 \mathrm{~km} \mathrm{~s}^{-1}\right.$; Riera, Phillips, \& Mampaso 1990; Trammell et al. 1993). The higher shock velocity derived by us is basically due to the higher [O III] flux obtained in our observations.

$[\mathrm{N} \mathrm{II}]$ and $[\mathrm{Fe}$ II] lines show the largest intensity contrast between the tips and the innermost regions of the lobes among forbidden transitions. [Fe II] lines are known to be good tracers of astrophysical shocks (in supernovae, Herbig-Haro objects, PNs, etc.): [Fe II] lines are very sensitive to the high densities and temperatures in the shocked gas; moreover, fast shocks are able to extract substantial amounts of Fe from dust grains (e.g., Welch et al. 1999; Reipurth et al. 2000). Therefore, the intensity enhancement of the $[\mathrm{Fe}$ II] (and [N II]) lines is very likely related to the presence of intense shocks in these regions. In addition, the strongest [Fe II] line emission is expected in regions where the compression and heating of the gas is largest, for example, at the head of a bow shock, where the velocity component normal to the shock is greatest. The presence of bow shocks at the tips of the lobes of CRL 618, where the maximum [Fe II] emission is observed, is suggested by bright, curved features in the direct $H S T$ images of the nebula (Fig. 1). Finally, the presence of intense [Fe II] lines in the shock-heated and compressed gas in the lobes of CRL 618 points to dissociative $\left(V_{s}>30 \mathrm{~km} \mathrm{~s}^{-1}\right) \mathrm{J}$-shocks rather than C-shocks (Reipurth et al. 2000), consistent with the relatively large shock velocity derived from diagnostic line ratios and the kinematics of the lobes (see $\S 7$ ).

The ionization fraction in the shocked lobes is significantly lower than in region $\mathrm{A}^{\prime}$ as suggested by the much larger [O I] $\lambda 6300 / \mathrm{H} \alpha$ ratio, $\sim 0.5-0.9$ (excluding scattered light), in the former. We discuss in detail the ionization in the lobes in $\S 9.2$.

\subsubsection{The Scattered-Light Halo}

A diffuse halo surrounding the bright optical lobes of CRL 618 is visible in the $\mathrm{H} \alpha$ direct images of the nebula (Figs. $1 b$ and $1 c$ ). The scattered feature $\mathrm{C}$ in the $\mathrm{H} \alpha$ spectra arises in this halo, and it is particularly noticeable in the region just beyond the tip of the east lobe. Accordingly, the $\mathrm{H} \alpha$ emission from this halo is very likely light (originally arising in the $\mathrm{H}$ II region) that escapes preferentially in the direction of the lobes and is scattered by the innermost parts of the AGB CSE. (The more distant regions of the AGB CSE are visible in molecular line emission; see $\S 1$.)

\section{THE INCLINATION OF THE NEBULA}

The presence of scattered features (C and $\mathrm{B})$ in the $\mathrm{H} \alpha$ spectrum of CRL 618 offers a valuable opportunity to derive the inclination of the nebula: by comparison of the redshift of the scattered emission with the intrinsic velocity of the dust (whenever these quantities can be determined).

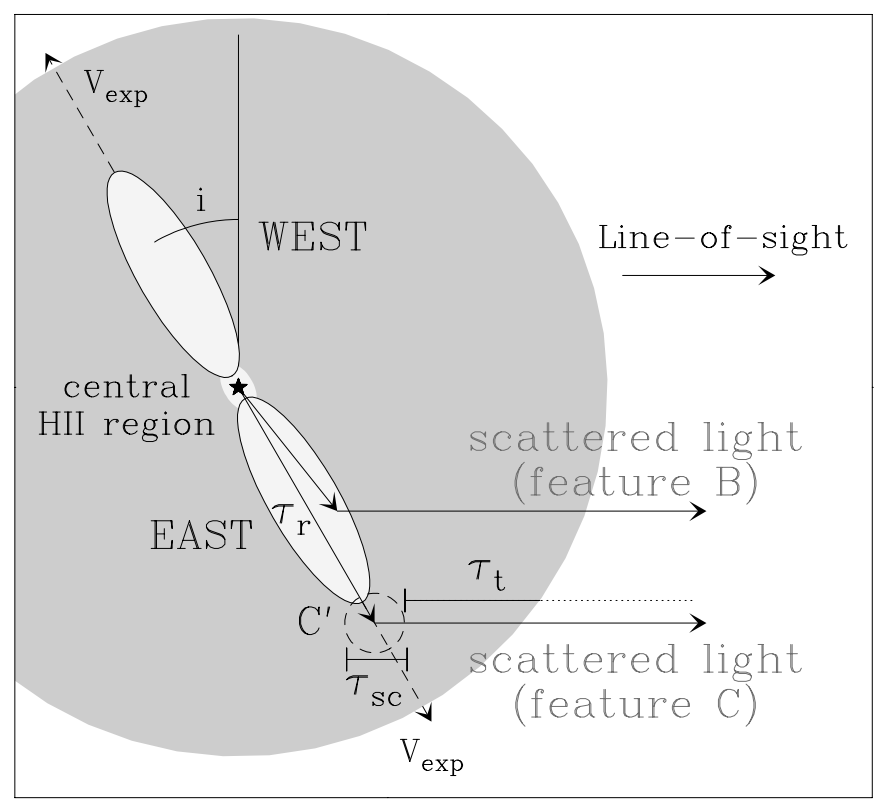

FIG. 7.- Schematic structure of CRL 618. The optical lobes and the central $\mathrm{H}$ II region are represented by white ellipses. The surrounding AGB envelope, which is radially expanding at velocity $V_{\text {exp }}$, is represented by the gray circle. The light arising in the $\mathrm{H}$ II region escapes preferentially through the lobes, illuminating a compact region beyond the east lobe tip (region $\mathrm{C}^{\prime}$ ) that scatters light into the line of sight leading to feature $\mathrm{C}$. The light from the $\mathrm{H}$ II region is also scattered by rapidly outflowing dust inside or in the walls of the lobes leading to feature B. The different optical depth components $\left(\tau_{r}, \tau_{t}\right.$, and $\tau_{\text {sc }}$; see $\left.\S 8.1\right)$ are indicated. We do not intend to accurately reproduce the actual shape or multiple lobe character of CRL 618 or the relative dimensions of the different nebular components. [See the electronic edition of the Journal for a color version of this figure.]

Features B and C are produced, respectively, by (1) fastmoving dust mixed with the atomic gas inside or in the walls of the lobes and (2) dust beyond the lobes, in the slowly expanding, extended envelope that surrounds the optical lobes (see $\S 5.1$ and Fig. 7). The limited spectral resolution in our spectra does not allow us to separately measure the redshift of features $\mathrm{B}$ and $\mathrm{C}$ when both components are simultaneously present (all along the east lobe up to offsets $\sim 8^{\prime \prime}$ ). However, at the tip of the east lobe, only feature $\mathrm{C}$ is observed in the $\mathrm{H} \alpha$ spectra, and the redshift of this feature can be accurately determined. By comparison of this redshift with the intrinsic expansion velocity of the dust in the AGB CSE producing feature C, $V_{\exp } \sim 17.5 \mathrm{~km} \mathrm{~s}^{-1}$ LSR $(\S 1)$, we can readily derive the nebular inclination.

The redshift of feature $\mathrm{C}$ is apparent in Figure 8 (left-hand panels), where we show its spectral profile derived from slits N93 and S93 integrating the long-slit spectra from offsets 8 ".5 to $11^{\prime \prime} 5$. The redshift of feature $\mathrm{C}$ for both slit positions has been obtained by fitting a Gaussian function to the core of the feature, which is roughly symmetric.

For $\mathrm{N} 93$ and $\mathrm{S} 93$, the redshift with respect to $V_{\text {sys }}$ (given by the Gaussian center) is $8 \pm 1.5$ and $6 \pm 1.5 \mathrm{~km} \mathrm{~s}^{-1}$, respectively. These values yield an inclination (with respect to the plane of the sky) of $\sim 27^{\circ} \pm 6^{\circ}$ (from slit N93) and $20^{\circ} \pm 5^{\circ}$ (from slit S93) for the dust reflecting the $\mathrm{H} \alpha$ photons ahead of the east lobe.

As we have shown, feature $\mathrm{C}$ beyond the east lobe has its counterpart in the weak halo seen in the $\mathrm{H} \alpha$ images of the nebula at the same position (Fig. 1). The orientation of this halo and the pure emission lobe in the plane of the sky is 

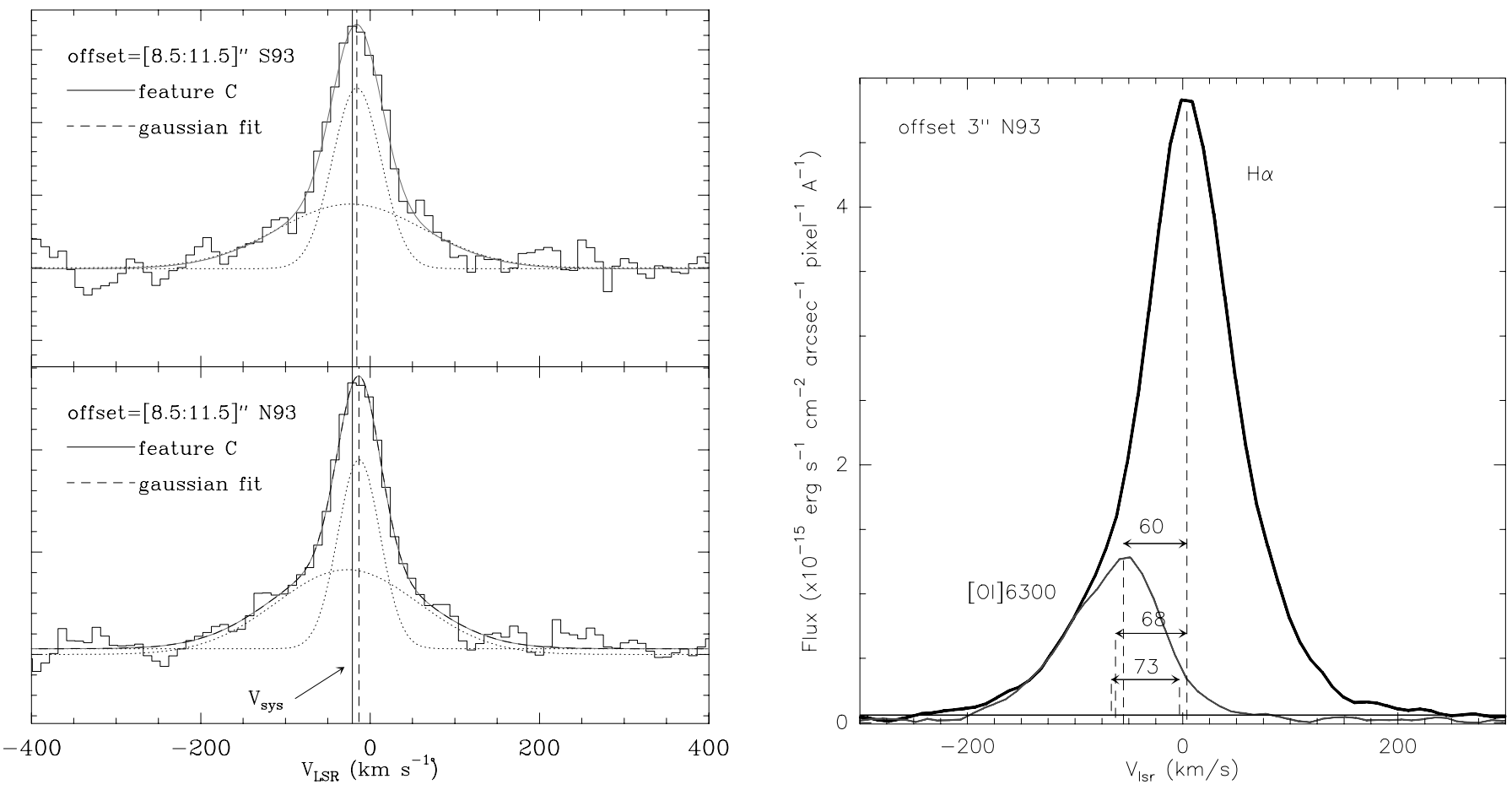

FIG. 8. - Left: Spectral profile of feature C at the tip of the east lobe (solid histogram); the H $\alpha$ long-slit spectra have been spatially averaged from offset 8".5 to 11".5 for slit positions N93 (bottom) and S93 (top). The core of feature C is roughly symmetric and has been approximated by a Gaussian function (dashed line). The center of the Gaussian (vertical dashed line) is redshifted with respect to the systemic velocity (vertical solid line). The wings of the feature have been also fitted by a Gaussian function. Right: One-dimensional $\mathrm{H} \alpha$ (thick line) and [O I] $\lambda 6300$ (thin line) spectra obtained at offset $3^{\prime \prime}$ for slit N93. The redshifted $\mathrm{H} \alpha$ emission, contrary to the blueshifted [ $\left.\mathrm{O}_{\mathrm{I}}\right]$ transition, is dominated by features $\mathrm{A}, \mathrm{B}$, and $\mathrm{C}$ (see text) rather than by emission locally produced by the shocked gas in the east lobe.

roughly the same. This is most likely due to light from the core escaping preferentially along the lobes of CRL 618 . Hence, we can expect the previous values of the inclination to be a relatively good representation of the mean nebular inclination (for an overall axial symmetry).

The different inclinations $\left(\Delta i=7^{\circ}\right)$ derived for feature $\mathrm{C}$ in slits S93 and N93 could indicate different orientations of the different lobe components along the line of sight, a likely possibility given their different orientations in the plane of the sky $\left(\triangle\right.$ P.A. $\left.\sim 15^{\circ}\right)$.

We can also derive $i$ by measuring the velocity of feature $\mathrm{B}\left(V_{\mathrm{B}}\right)$ with respect to the local emission component ( $V_{\text {local }}$, obtained from the forbidden lines) at the same position. $V=V_{\mathrm{B}}-V_{\text {local }}$ is a direct measurement of the intrinsic expansion velocity of the lobes; therefore, $i=\sin ^{-1}\left(V_{\text {local }}-V_{\text {sys }}\right) /\left(V_{\mathrm{B}}-V_{\text {local }}\right)$. From our spectra, we can derive only a lower limit to the redshift of feature B because the $\mathrm{H} \alpha$ profile also has important contributions from features $\mathrm{A}$ and $\mathrm{C}$ at the position where feature $\mathrm{B}$ is observed (offset $\sim 3^{\prime \prime}$ ). This leads to a combined $\mathrm{H} \alpha$ profile with a smaller redshift than that of feature B alone and, therefore, only a lower limit to the intrinsic expansion velocity of the lobes. The lower limit for $V$ translates into an upper limit for $i$.

In the right-hand panel of Figure 8, we show $\mathrm{H} \alpha$ and [O I] $\lambda 6300$ one-dimensional spectra at the position where the scattered continuum and feature $B$ reach maximum intensity in the east lobe for slit N93. The velocities of the $\mathrm{H} \alpha$ and [O I] lines measured (with uncertainties of $\pm 1.5 \mathrm{~km} \mathrm{~s}^{-1}$ ) at the peak are $V_{\mathrm{B}}=5 \mathrm{~km} \mathrm{~s}^{-1}, V_{\text {local }}=-55 \mathrm{~km} \mathrm{~s}^{-1}$, which yields $V>60 \mathrm{~km} \mathrm{~s}^{-1}$ (at this position) and $i<34^{\circ}$. If, instead of using the line peaks for the velocity measurements, we use the centroids of the FWHM $\left(V_{\mathrm{B}}=5 \mathrm{~km} \mathrm{~s}^{-1}\right.$, $\left.V_{\text {local }}=-63 \mathrm{~km} \mathrm{~s}^{-1}, V>68 \mathrm{~km} \mathrm{~s}^{-1}\right)$ or the centroids of the full width at a $3 \sigma$ level $\left(V_{\mathrm{B}}=-6 \mathrm{~km} \mathrm{~s}^{-1}, V_{\text {local }}=-67 \mathrm{~km}\right.$ $\mathrm{s}^{-1}, V>73 \mathrm{~km} \mathrm{~s}^{-1}$ ), we get values of $i<38^{\circ}$ and $39^{\circ}$, respectively.

The mean value of and upper limit to the inclination derived above are smaller than the inclination previously obtained by CS82. These authors obtain $i=45^{\circ}$, based on the analysis of the $\mathrm{H} \alpha$ scattered component produced by the fast dust within the lobes. We believe that this difference can be due to several reasons. First, the value given by CS 82 for the intrinsic speed of the outflow, $80 \mathrm{~km} \mathrm{~s}^{-1}$, is really a lower limit to $V$ because of the presence of multiple components in the $\mathrm{H} \alpha$ profile (as explained above). Second, the value for the radial velocity difference between the east and west lobes (which is equal to $2 V \sin i$ ) measured by CS82 from the forbidden line emission (e.g., [N II] $\lambda \lambda 6548,6583$, [S II] $\lambda \lambda 6716$, $6731), 114 \mathrm{~km} \mathrm{~s}^{-1}$, is most likely an overestimate. This is because the radial velocity difference between the lobes is not constant along their length (see Figs. 2 and 4 and Table 3). In particular, the $114 \mathrm{~km} \mathrm{~s}^{-1}$ velocity difference occurs only at the tips of the lobes (at offsets $\pm 6^{\prime \prime}$ ). But in the region where the most intense $\mathrm{H} \alpha$ scattered emission is observed (offset $\sim 3^{\prime \prime}$ ), coincident with the position where CS82 measure the redshift of the $\mathrm{H} \alpha$ scattered component, we observe a smaller radial velocity difference, $\sim 90 \mathrm{~km} \mathrm{~s}^{-1}$. Thus, if we use a larger value of the outflow speed $(>80 \mathrm{~km}$ $\left.\mathrm{s}^{-1}\right)$ and a smaller radial velocity difference $\left(\sim 90 \mathrm{~km} \mathrm{~s}^{-1}\right)$ in the CS82 method, we get an upper limit to the inclination of $34^{\circ}$, which is consistent with our previous estimates. 
TABLE 3

Velocity Data From the [O i] $\lambda 6300$ Line

\begin{tabular}{|c|c|c|c|c|c|c|}
\hline \multirow[b]{2}{*}{$\begin{array}{l}\text { OFFSET } \\
(\operatorname{arcsec})\end{array}$} & \multicolumn{3}{|c|}{ SLIT N93 } & \multicolumn{3}{|c|}{ SLIT S93 } \\
\hline & $\begin{array}{c}V_{\mathrm{LSR}}+21 \\
\left(\mathrm{~km} \mathrm{~s}^{-1}\right)\end{array}$ & $\begin{array}{l}\mathrm{FWHM}^{\mathrm{a}} \\
\left(\mathrm{km} \mathrm{s}^{-1}\right)\end{array}$ & $\begin{array}{c}\mathrm{FWZI}^{\mathrm{b}} \\
\left(\mathrm{km} \mathrm{s}^{-1}\right)\end{array}$ & $\begin{array}{c}V_{\mathrm{LSR}}+21 \\
\left(\mathrm{~km} \mathrm{~s}^{-1}\right)\end{array}$ & $\begin{array}{c}\mathrm{FWHM}^{\mathrm{a}} \\
\left(\mathrm{km} \mathrm{s}^{-1}\right)\end{array}$ & $\begin{array}{c}\mathrm{FWZI}^{\mathrm{b}} \\
\left(\mathrm{km} \mathrm{s}^{-1}\right)\end{array}$ \\
\hline \multicolumn{7}{|c|}{ West (Weak) Lobe } \\
\hline$-6.2 \ldots \ldots \ldots \ldots$ & 76 & 125 & 220 & 56 & 136 & 190 \\
\hline$-3.7 \ldots \ldots \ldots \ldots$ & 56 & 75 & 190 & 44 & 73 & 190 \\
\hline$-1.0 \ldots \ldots \ldots \ldots$ & 61 & 61 & 90 & 56 & 61 & 100 \\
\hline \multicolumn{7}{|c|}{ East (Bright) Lobe } \\
\hline $1.0 \ldots \ldots \ldots \ldots$ & -49 & 150 & 200 & -60 & 125 & 170 \\
\hline $3.2 \ldots \ldots \ldots \ldots$ & -31 & 110 & 250 & -45 & 125 & 240 \\
\hline $5.4 \ldots \ldots \ldots \ldots$ & -39 & 117 & 230 & -54 & 146 & 230 \\
\hline $6.5 \ldots \ldots \ldots \ldots$ & -69 & 138 & 210 & -64 & 177 & 220 \\
\hline
\end{tabular}

a Deconvolved with the spectral resolution, $\sim 50 \mathrm{~km} \mathrm{~s}^{-1}$.

b Measured at a $\sim 3 \sigma$ level.

Finally, a low mean value of the inclination is in better agreement with the similar average extinction deduced for the east and west lobe (see $\S 8)$.

\section{KINEMATICS}

\subsection{The Shocked Lobes}

In order to study the kinematics in the lobes of CRL 618, we have chosen the most intense forbidden lines, which originate in the lobes and have profiles uncontaminated by scattered-light components. Since the [N II] lines have also some contribution from reflected light (Trammell et al. 1993 found that the polarization of these lines is $\sim 6 \%$ ), we have based most of our analysis on the intense, unpolarized [O I] and $[\mathrm{S}$ II] lines.

The [O I] and [S II] profiles along the slit N93 for the west and east lobe are approximately point symmetric with respect to the spatial origin and systemic velocity (Fig. 2), suggesting that they have probably a similar kinematical structure. This result is not in principle expected from the different morphology of the east and west lobes. The projected radial velocity gradient is not constant in either lobe. In the innermost parts of the lobes the projected velocity decreases with the distance from the nebular center, this behavior being more pronounced in the east lobe (Table 3 ). In the outer parts of the lobes, the velocity increases with the distance, reaching projected velocities (with respect to $V_{\text {sys }}$ ) up to 70 and $80 \mathrm{~km} \mathrm{~s}^{-1}$ at the tip of the east and west lobe, respectively. In these regions, the emission arises in the compact, bow-shaped emitting knots at $\pm 6^{\prime \prime}$. Considering the low inclination for the nebula derived in $\S 6, i \sim 24^{\circ}$, the lobes of CRL 618 are expanding with velocities up to $\sim 200$ $\mathrm{km} \mathrm{s}^{-1}$ (at the tips). The changing radial velocity gradient observed in our low angular resolution (ground-based) spectra may result from superposition of multiple kinematical components as suggested by the complex morphology and small-scale $\left(\sim 0{ }^{\prime \prime} 2\right)$ structure seen in the HST images. Of course, it is also possible that there are acceleration and deceleration processes operating in the lobes.

The FWHM of the forbidden lines along the lobes also increases outward, from $60-75 \mathrm{~km} \mathrm{~s}^{-1}$ closer to the nebula center to $\sim 150 \mathrm{~km} \mathrm{~s}^{-1}$ at the lobe tips (Table 3). The large line widths measured at the tips of the lobes (Fig. 9) are consistent with the bow-shaped features being regions accelerated by bow shocks: the different orientations of the velocity vectors in a bow shock structure produce a large velocity dispersion (see, e.g., HRH87). The total width (at zero intensity level) of the line does not strongly vary along the lobes but rather is fairly constant with a mean value of $\sim 200 \mathrm{~km} \mathrm{~s}^{-1}$.

The spectral profile of any low-excitation emission line from a bow shock can be used to estimate the shock velocity $V_{s}$. The shock velocity equals the full width (at zero intensity) of the line for radiating bow shocks, independent of orientation angle, preshock density, bow shock shape, and preshock ionization stage (HRH87). We derive $V_{s}=200-230 \mathrm{~km} \mathrm{~s}^{-1}$ for the bow shocks seen at the tips of the east and west lobes. This value agrees well with the value

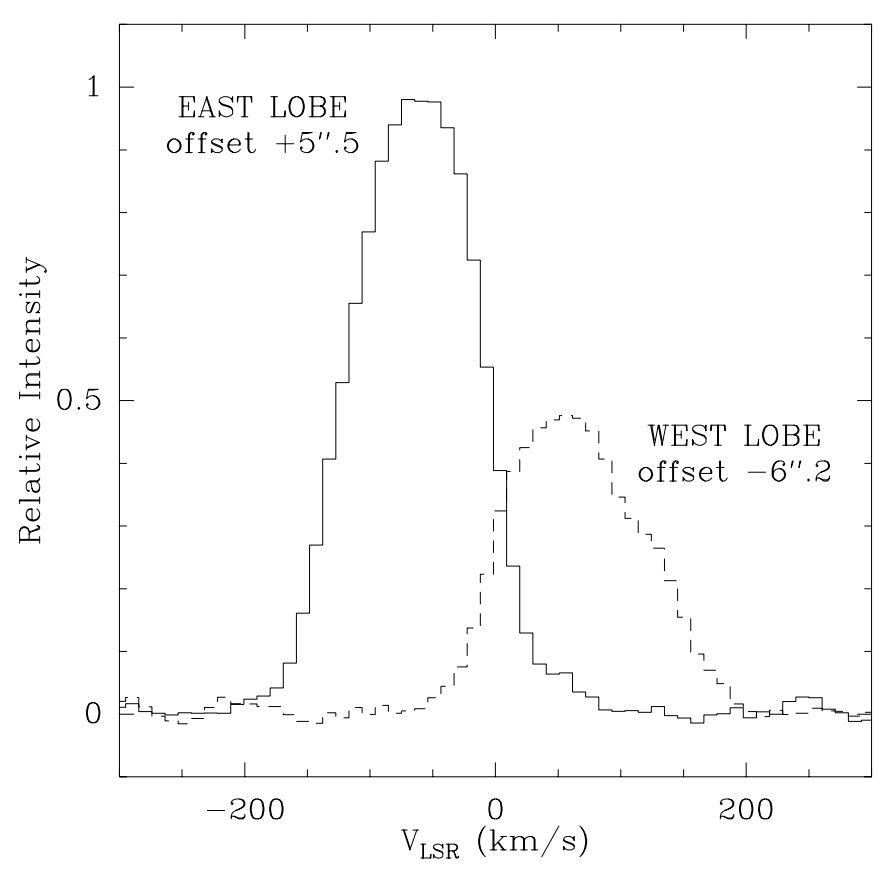

FIG. 9.- Spectral profile of the [O I] $\lambda 6300$ line for slit N93 at the tips of the east and west lobes, coincident with the bow-shaped emitting regions. 
of $V_{s}$ inferred directly from the projected velocities of these regions assuming an inclination of $24^{\circ}$. This result therefore supports the low inclination of the nebula we have derived (§ 6). In addition, the centrally peaked profiles in Figure 9 are consistent with the predictions for bow shock models by HRH87 with $i<25^{\circ}$ (for larger inclinations the model profiles show two well-separated peaks).

The shock velocity derived from the observed line ratios and their comparison with predictions of bow shock excitation models by HRH87 $\left(V_{s}<90 \mathrm{~km} \mathrm{~s}^{-1} ; \S 5.2 .3\right)$ is smaller than that directly derived from the line profile. Similar differences are also present for many Herbig-Haro objects observed and modeled by these authors suggesting that the existing models (necessarily simplistic) cannot accurately reproduce the complex excitation and spatiokinematical structure of the shocked material. Moreover, the preshock density in the HRH87 models, $300 \mathrm{~cm}^{-3}$, is very likely lower than the actual value (see $\S 10$ ). The shock velocity obtained from the line profile is probably less affected by errors in the model, since it arises only from geometrical considerations, and may better represent the actual velocity of the shock. Only if the line emission from the shocked post-AGB wind itself (which is moving faster than the forward shocks) was significant compared to that from the shocked AGB ambient material (unlikely to be the case in CRL 618; C.-F. Lee $\&$ R. Sahai 2002, in preparation) would the line FWZI be an upper limit to $V_{s}$. $V_{s}$ derived from the line ratios depends on poorly known parameters like, e.g., the presence and strength of magnetic fields. Models with higher magnetic fields require substantially larger shock velocities to reproduce the same line ratios (Hartigan et al. 1994). For example, for a preshock density $\gtrsim 10^{5} \mathrm{~cm}^{-3}(\S 10)$, magnetic fields of $\sim 30 \mu \mathrm{G}$ are required to reproduce the dereddened [N II] $\lambda 6583 /\left[\mathrm{O}_{\mathrm{I}}\right] \lambda 6300$ ratio $(\sim 0.5$; see Table 1 and $\S 8)$ for a planar shock with speed $V_{s}=90 \mathrm{~km} \mathrm{~s}^{-1}$. To produce the same ratio when $V_{s}=200 \mathrm{~km} \mathrm{~s}^{-1}$, the required magnetic field is much larger $(>3000 \mu \mathrm{G})$.

Finally, from a comparison of the spectra obtained for slits N93, S93, and PA3, we infer some differences in the kinematics of the different lobe components (note that each lobe of CRL 618 consists of at least two well-separated features; Fig. 2). For example, the south subcomponent of the east lobe (better probed by slit S93) is expanding 5-20 km $\mathrm{s}^{-1}$ faster than the north one. Similarly, the northern subfeature of the west lobe (better seen in slit N93) is $5-20 \mathrm{~km} \mathrm{~s}^{-1}$ faster than the southern. These differences could be due to different absolute ejection velocities or inclinations of the lobe components. We note also that, in general, N93 and S93 provide similar FWHMs for the west lobe, but for the east lobe, the largest FWHMs are measured from slit S93, where the line FWHM reaches up to $\sim 180 \mathrm{~km} \mathrm{~s}^{-1}$. The larger widths in the southeast lobe subcomponent are unlikely to be related to projection effects and must be due to intrinsic differences in the kinematics.

\subsection{Region $A^{\prime}$ and the Inner $\mathrm{H}$ II Region}

From the analysis of the $\mathrm{H} \alpha$ profile we can also obtain valuable information on the kinematics in regions of the CRL 618 nebula other than the shocked lobes. The presence of the blueshifted, intense $\mathrm{H} \alpha$ emission component in the east lobe close to the nebula center (feature A; Fig. 2) indicates ionized gas moving away at moderate velocity (15-30 $\mathrm{km} \mathrm{s}^{-1}$ ) from the central star. We have measured the veloc- ity of this feature from the [N II] lines, which are less contaminated by scattered light (note that the blueshift of feature A in the $\mathrm{H} \alpha$ spectra is masked by superposition with the intense, redshifted feature $\mathrm{B}$ and probably also feature $\mathrm{C}$ ).

The kinematics of the $\mathrm{H}$ in region cannot be so straightforwardly obtained from our data, since the emission from this region is seen only after being reflected by the dust (through features $\mathrm{B}$ and $\mathrm{C}$ ); therefore, the resulting line profile is affected by the distribution and kinematics of the dust. The FWHM of feature C, observed at the tip of the lobes (Fig. 8) and deconvolved with the spectral resolution of our data, is $\sim 60 \mathrm{~km} \mathrm{~s}^{-1}$, suggesting an expansion velocity for the gas in the $\mathrm{H}$ II region of $\sim 30 \mathrm{~km} \mathrm{~s}^{-1}$. This figure has to be considered as an upper limit, since the reflecting dust could show a range of inclinations resulting from the opening angle of the lobes (up to $15^{\circ}$ according to the lobe aperture in the plane of the sky). Our result is consistent with the low expansion velocity of the $\mathrm{H}$ II region measured from the profile of $\mathrm{H}$ radio recombination lines $\left(20 \mathrm{~km} \mathrm{~s}^{-1}\right.$; Martín-Pintado et al. 1988). The weak wings of feature $\mathrm{B}$ in the $\mathrm{H} \alpha$ and $\mathrm{H} \beta$ lines extend up to LSR velocity $\sim 240 \mathrm{~km} \mathrm{~s}^{-1}$ (at a level of $5 \sigma$ ). Such extended red wings are most likely the result of the large velocities of the reflecting grains within the lobe (up to $\sim 200 \mathrm{~km} \mathrm{~s}^{-1}$ ) and also partially to the lobe opening angle; however, the presence of rapidly outflowing material in the $\mathrm{H}$ II region cannot be ruled out.

\section{EXTINCTION}

We have estimated the relative variation of the extinction along the lobes of CRL 618 by comparing the $\mathrm{H} \alpha$ and $\mathrm{H} \beta$ spatial profiles along the axis (from slit position N93). The two long-slit spectra have been aligned in the direction of the slit using the west lobe brightest knot (at the tip of the lobe) as our spatial reference. We estimate that errors in the alignment are $\lesssim 1$ pixel $(\lesssim 0$ "! 2$)$. The uncertainty in the positions of the slits used for obtaining the $\mathrm{H} \alpha$ and $\mathrm{H} \beta$ spectra in the direction perpendicular to the slit is a few pixels and, therefore, smaller than the seeing (1".6; $\S 2.2)$. The spatial distribution for both transitions and the adjacent continuum was obtained integrating our long-slit spectra in the spectral direction. In order to derive the Balmer decrement, the continuum emission was first subtracted from the $\mathrm{H} \beta$ spatial profile but not from the much brighter $\mathrm{H} \alpha$ emission (in this case, the continuum contribution is negligible, $\lesssim 1 \%$ ).

The spatial distribution of the extinction derived from the $\mathrm{H} \alpha / \mathrm{H} \beta$ ratio is shown in Figure 10 (top panel). In this figure, the optical depth at $4861.3 \AA$, $\tau_{4861}$, has been calculated assuming the Galactic reddening curve by Howarth (1983) and an intrinsic Balmer decrement of 3. This value of the Balmer decrement allows easy comparison of our results with previous estimates of the extinction, which usually assume $\mathrm{H} \alpha / \mathrm{H} \beta=3$. Moreover, the previous value is expected for high-velocity shocks like those in CRL 618 (§7), for which the intrinsic Balmer ratio approaches the recombination value (collisional excitation increases the intrinsic Balmer ratio only for low-velocity shocks, less than $70 \mathrm{~km} \mathrm{~s}^{-1}$; Hartigan et al. 1994). The conversion from $\tau_{4861}$ to extinction in magnitudes in the $V$ band, $\tau_{4861}=0.93 A_{V}$, has been derived using the extinction law parametrization by Cardelli, Clayton, \& Mathis (1989) and assuming the ratio of total to selective absorption, $R_{V}$, equal to 3.1. The error bars in Figure 10 are statistical and do not account for 


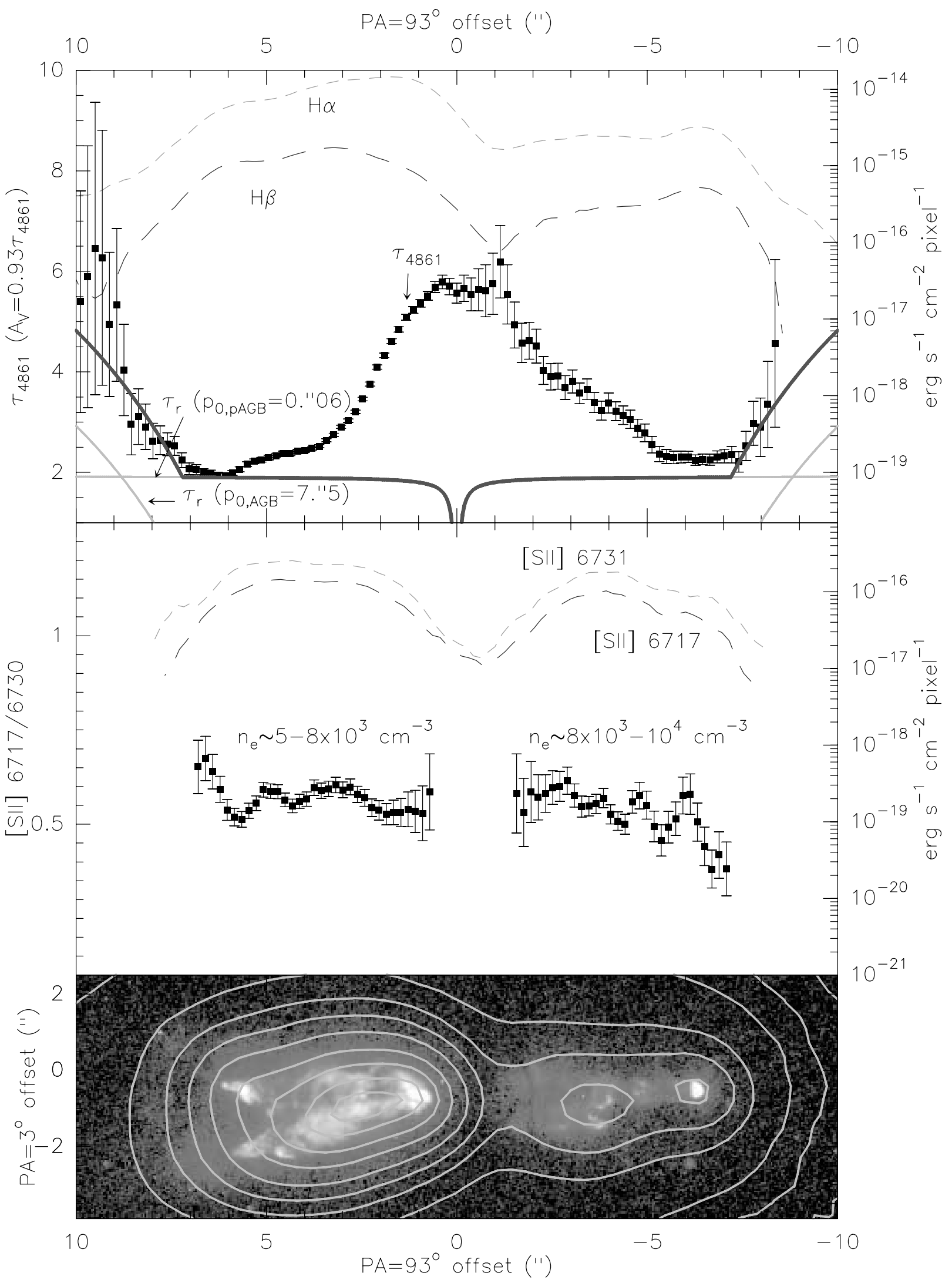

FIG. 10.-Top: Spatial variation along slit N93 of the optical depth at $4861 \AA$ (black squares), derived from the $\mathrm{H} \alpha$-to- $\mathrm{H} \beta$ relative flux (the $\mathrm{H} \alpha$ and $\mathrm{H} \beta$ spatial profiles are drawn using dashed lines). Thin solid lines represent the radial optical depth, $\tau_{r}$, due to dust in the AGB CSE and the post-AGB wind (see $\S 8$ ). The thick line is the sum of these two optical depths. Middle: [S II] $\lambda 6716 / \lambda 6731$ doublet ratio, which is an indicator of the electron density, along N93 (black squares). The spatial profiles of the [S II] $\lambda \lambda 6716,6731$ doublet (dashed lines) are also shown. Bottom: HST (gray scale) and ground-based (contours) images of CRL 618. [See the electronic edition of the Journal for a color version of this figure.] 
systematic errors (e.g., arising from an incorrect value of the intrinsic Balmer decrement, absolute flux calibration, or small misalignments between the $\mathrm{H} \alpha$ and $\mathrm{H} \beta$ spectra).

The $\mathrm{H} \alpha / \mathrm{H} \beta$ ratio clearly varies along the nebular axis (Fig. 10). This variation is most likely not due to a change of the intrinsic Balmer ratio, since we do not expect the shock characteristics (e.g., shock speed and preshock density) to vary sufficiently along the nebular axis. Neither the velocity of the shocks nor the electron density shows systematic variations along the lobes (see $\S \S 7$ and 9). Accordingly, the observed variation of the $\mathrm{H} \alpha / \mathrm{H} \beta$ ratio must be related to extinction by nebular dust.

The nebular extinction varies along the axis of CRL 618 as follows: the maximum extinction is produced close to the nebular center and then gradually decreases toward the outermost parts of the lobes. The extinction in the innermost nebular regions is particularly uncertain and is very likely underestimated by a large factor. In fact, from the $H S T$ images we can see that the emission from the $2^{\prime \prime}$ region around the nebular center is well below the noise, suggesting a very high obscuration there (see Fig. 1). We deduce that the extinction toward the central star of CRL 618 is $A_{V}>10 \mathrm{mag}$, given the upper limit to the stellar continuum level estimated from the R1200Y spectra, $\$ 2.5 \times 10^{-16} \mathrm{ergs}$ $\mathrm{s}^{-1} \mathrm{~cm}^{-2} \AA^{-1}$, and the expected flux from a $30,000 \mathrm{~K}$ blackbody, $2.5 \times 10^{-12} \mathrm{ergs} \mathrm{s}^{-1} \mathrm{~cm}^{-2} \AA^{-1}$. Our low-resolution, ground-based $\mathrm{H} \alpha$ and $\mathrm{H} \beta$ spectra, used to calculate the extinction, show some weak emission from the innermost nebular region. However, this is a result of the poor spatial resolution in these spectra, which spreads out some light from nearby nebular regions in the lobes (where the extinction is smaller) into the nebular center (with, very likely, much larger extinction). The result is an underestimate of the extinction in these inner regions as deduced from the observed Balmer decrement.

Beyond the central region, the extinction decreases with the distance from the nebular center reaching a relative minimum around offsets of roughly \pm 6 ". 5 . At offsets larger than $\pm 7^{\prime \prime}$ (beyond the tips of the lobes) the extinction increases again.

We note that the extinction curve along the nebula is quite symmetric; i.e., the extinction is similar for the east and west lobe: the weak west lobe appears on average only about $15 \%$ more extinguished than the bright east lobe. The difference is even smaller at the tip of the lobes. This result indicates that the lower $\mathrm{H} \alpha$ surface brightness for the west lobe compared to the east (by a factor of $\sim 5$ ) is intrinsic and not due (at least totally) to a much larger extinction of the receding lobe. In fact, most of the $\mathrm{H} \alpha$ emission from the east lobe arises from region $\mathrm{A}^{\prime}$, which does not have a counterpart in the west lobe. The similar extinction for the east and west lobe is consistent with the similar brightness of both lobes for most forbidden lines (e.g., [O I], [S II]), for which the east lobe is only a factor of $\sim 1.5$ brighter than the west lobe. Finally, the similar extinctions derived for the east and west lobe suggest a low inclination of the nebular axis with respect to the plane of the sky (assuming that most of the extinction is produced by the AGB CSE surrounding the lobes; $\S 1$ ), consistent with the value we derive from the analysis of the scattered $\mathrm{H} \alpha$ emission $\left(i \sim 24^{\circ} ; \S 6\right)$.

Our results on the extinction in CRL 618 are in good agreement with previous estimates using the Balmer decrement method: Kelly et al. (1992) derive an average extinction in the nebula of $A_{V}=4.5 \pm 0.2$, which is within the nebular extinction range derived by us (Fig. 10). From the analysis of [S II] $\lambda \lambda 6716,6731$ and [S II] $\lambda \lambda 4069,4076$ lines, these authors also found that both the east and west lobes have similar extinction values, $A_{V}=2.1 \pm 0.2$ and $2.9 \pm 0.3 \mathrm{mag}$, respectively.

\subsection{Effect of the Scattering on $A_{V}$ : Radial Extinction beyond the Lobes}

The estimate of the absolute extinction along the line of sight from the Balmer decrement in an object like CRL 618 is problematic mainly because of the contribution of scattered light to the total flux of the $\mathrm{H}$ i recombination lines, as already noticed by, e.g., Trammell et al. (1993) and Kelly et al. (1992). The effects of such contribution on the value of the observed $\mathrm{H} \alpha / \mathrm{H} \beta$ ratio are difficult to quantify: on the one hand, the scattering will produce an "artificial" decrease of the observed $\mathrm{H} \alpha / \mathrm{H} \beta$ ratio due to the highest reflection efficiency for blue photons; on the other hand, the extinction of the light from the innermost regions, where it originates, to the regions where it is scattered by the dust (radial extinction) will produce reddening that may or may not compensate the previous artificial increase of the $\mathrm{H} \alpha / \mathrm{H} \beta$ ratio.

The presence of nebular dust is expected to affect not only the absolute value of the extinction but also its relative variation from one region to another in the nebula. In regions where both local and scattered $\mathrm{H} \alpha$ and $\mathrm{H} \beta$ emission is present, the optical depth derived from the Balmer decrement, $\tau$, is not only the line-of-sight or tangential optical thickness, $\tau_{t}$ (from the lobes to the observer), as desired, but a complex function of $\tau_{t}$, the radial optical depth $\tau_{r}$ (from the star up to the lobes where the light is being reflected), and the relative intensity of the Balmer emission locally produced within the lobes and in the inner $\mathrm{H}$ II region. All these quantities will vary along the nebula in a different way, and they will combine to yield the extinction profile in Figure 10.

At the tip of the CRL 618 east lobe (and tentatively in the tip of the west lobe) only $\mathrm{H} \alpha$ emission from feature $\mathrm{C}$ (and "C?") is observed; i.e., the Balmer emission is dominated by the scattered component (Fig. 4). In a region like this, where the local emission is negligible with respect to the scattered component, $\tau$ only depends on $\tau_{r}$ and $\tau_{t}$ in a relatively simple way:

$$
\tau=\tau_{t}+\tau_{r}-\ln \tau_{\mathrm{sc}},
$$

where $\tau_{\mathrm{sc}}$ is the scattering optical thickness of the small region at the tip of the east lobe that produces feature $\mathrm{C}$ (region $\mathrm{C}^{\prime}$; see Fig. 7) and the optical depths $\tau_{r}$ and $\tau_{t}$ include both scattering and absorption. This equation is valid if the scattering and absorption optical depths in region $C^{\prime}$ are small $(\ll 1)$, as expected beyond the tips of the lobes. In this case, $\tau_{t}$ is mostly due to foreground dust (not directly illuminated by the central source) between region $\mathrm{C}^{\prime}$ and the Earth. Using this equation, we analyze the spatial variation of the extinction in Figure 10 to study the structure of the lobes. At the tips of, and beyond, the lobes (offsets $\gtrsim 7^{\prime \prime}$ ), the extinction increases with the distance from the nebular center (Fig. 10). This increase, which is not expected a priori given the progressive decrease of $\tau$ with distance for inner regions, can be explained if $\tau \approx \tau_{r}$ in this region, i.e., if the radial extinction is larger than the tangential component. In fact, the tangential optical depth will decrease (not increase) with the distance from the central 
star in the plane of the sky, $p$, for any reasonable density law for the outer CSE [e.g., $\tau_{t} \propto p^{-1}$ for a density law $\left.\rho=\rho_{0}\left(r_{0} / r\right)^{2}\right]$. However, the radial extinction is expected to increase with $p$, as observed, for any reasonable density law within the lobes.

We have computed the radial extinction as a function of the observed spatial offset, $p=r \cos i$, for two different density laws within the lobes of CRL 618: (1) $\rho=\rho_{0}\left(r_{0} / r\right)^{2}$, which leads to $\tau_{r} \propto\left(1 / p_{0}-1 / p\right)$; and (2) $\rho=$ const, which yields $\tau_{r} \propto p-p_{0}$. The increasing extinction beyond offset $7^{\prime \prime}$ can be reproduced for both laws (note that, in fact, for $p \sim p_{0}$, the radial extinction $\tau_{r}$ in case 1 can be approximated by a straight line, which corresponds to case 2) but always requires values of $p_{0} \gtrsim 7^{\prime \prime}$. (We will not discuss case 2 further because $\rho \propto r^{-2}$ best represents the density in most AGB CSEs.) A small $p_{0}\left(\ll 7^{\prime \prime}\right)$ results in the extinction reaching a constant value $\left(\tau_{r} \propto 1 / p_{0}\right)$ very quickly (Fig. 10), which is inconsistent with the data.

The large $p_{0}$ obtained implies that there is a substantial component of the radial extinction that is produced by dust outside of the lobes (i.e., in the AGB CSE). We can only set an upper limit to the component of the radial extinction produced by dust inside the lobes (presumably filled by the post-AGB wind), based on the minimum value of $\tau_{4861}$ (about 2) obtained at the tips of the lobes (offsets of \pm 6.5 ). Both the above components (referred to as $\tau_{\mathrm{AGB}}$ and $\tau_{\mathrm{pAGB}}$ ) and their sum are shown in Figure 10.

\subsection{The Density Contrast between the $A G B$ and Post-AGB Wind}

Assuming that the gas filling the lobes is the post-AGB wind, we now derive $\rho_{\mathrm{pAGB}} / \rho_{\mathrm{AGB}}$ by comparing $\tau_{\mathrm{AGB}}$ and $\tau_{\mathrm{pAGB}}$. For a stationary outflow the mass-loss rate per solid angle is $\dot{M}_{\Omega} \equiv d \dot{M} / d \Omega=r^{2} \rho V$, and the corresponding radial optical thickness is

$$
\tau_{r} \propto \int_{r_{0}}^{r} n_{\text {dust }} d r=\kappa \frac{\dot{M}_{\Omega}}{V}\left(\frac{1}{r_{0}}-\frac{1}{r}\right),
$$

where $r$ is the distance from the star, $r_{0}$ is the inner radius, $\rho$ is the gas mass density, $V$ is the wind expansion velocity, and $\kappa$ depends on the grain properties and the gas-to-dust mass ratio. Combining equation (2) and the expression for $\dot{M}_{\Omega}$ above, we derive that

$$
\rho \propto \frac{\dot{M}_{\Omega}}{r^{2} V} \propto \frac{\tau_{r}}{r^{2}\left[\left(1 / r_{0}\right)-(1 / r)\right]} .
$$

From our fitting to the radial optical depths ( $\S 8.1$ ), which gives for the AGB CSE inner radius $r_{0, \mathrm{AGB}} \gtrsim 7.15$, and adopting for the post-AGB wind inner radius $r_{0, \mathrm{pAGB}} \sim 0$ ".06 (from the circumstellar dust model by Knapp, Sandell, \& Robson 1993), we obtain

$$
\frac{\rho_{\mathrm{AGB}}}{\rho_{\mathrm{pAGB}}} \gtrsim 600\left(\frac{0 ! .06}{r_{0, \mathrm{pAGB}}}\right) \frac{\kappa_{\mathrm{pAGB}}}{\kappa_{\mathrm{AGB}}},
$$

where the lower limit arises from the upper limit to $\tau_{\mathrm{pAGB}}$. The density ratio above depends on the poorly known inner radius of the post-AGB wind as well as on the unknown but expected differences in the dust-to-gas ratio and dust grain properties in the AGB CSE and in the fast post-AGB wind. Moreover, the intrinsic $\rho_{\mathrm{AGB}} / \rho_{\mathrm{pAGB}}$ ratio is likely to be lower because the region beyond the lobe tips is overdense compared to the original, unshocked, AGB CSE (since it contains material swept up by the post-AGB wind). The AGB-to-pAGB density ratio would also be significantly lower if the gas-to-dust ratio in the post-AGB wind was much higher than that in the AGB CSE, perhaps as a result of inefficient grain formation in the post-AGB wind emanating from the vicinity of a $30,000 \mathrm{~K}$ star.

Finally, as argued earlier, for offsets less than $7^{\prime \prime}$ the radial and tangential optical depth cannot be separately quantified from $\tau_{4861}$. The smooth decrease of $\tau_{4861}$ with distance from the central star may suggest that the derived extinction is dominated by the tangential component (as shown above, the radial component would increase or remain constant with the distance from the star). This is, in particular, most likely true for the west lobe, for which the fraction of scattered light in the $\mathrm{H} \alpha$ and $\mathrm{H} \beta$ emission lines is small. The similarity between the extinction profile for the east and west lobe as well as the expected lower extinction derived for the east (approaching) lobe again suggest that $\tau_{4861}$ is probably dominated by the tangential component. However, the derived extinction, which may still be affected by the scattering, does not allow us to perform a detailed modeling of $\tau_{t}$ to study the outer AGB CSE.

\section{DENSITY AND NEBULAR MASS}

\subsection{The Electron Density Distribution}

We have used the $[\mathrm{S}$ II] $\lambda 6716 / \lambda 6731$ doublet ratio to estimate the electron density, $n_{e}$, along the shocked lobes of CRL 618. The spatial profiles along the nebula axis (slit N93) for the two doublet lines and their ratio are plotted in Figure 10 (middle panel). We only show the ratios wherever the line intensities are over $3 \sigma$. There are no alignment errors to contend with since the two lines of the doublet were observed simultaneously.

Within the observational errors, the $[\mathrm{S}$ II] $\lambda 6716 / \lambda 6731$ ratio does not present a systematic variation along the east or west lobes, with mean values of 0.55 and 0.50 , respectively. The same average values are obtained for slit S93. Considering the [S II] $\lambda 6716 / \lambda 6731$ ratio versus electron temperature and density contour plots by Cantó et al. (1980) and an average electron temperature $T_{e}=10,000 \mathrm{~K}$ (§ 1), we derive densities for the east (west) lobe of $\sim(5-8) \times 10^{3}\left(\sim 8 \times 10^{3}-10^{4}\right) \mathrm{cm}^{-3}$. These figures are close to the critical densities for the $[\mathrm{S} \mathrm{II}] \lambda \lambda 6716,6731$ transitions; however, the good agreement between our results and previous estimates using different diagnostic lines (Kelly et al. 1992) suggests that collisional de-excitation of the $[\mathrm{S} \mathrm{II}]$ lines is not critical, at least, for most regions in the lobes.

There are some nonsystematic variations of the [S II] doublet ratio within the lobes that seem to be larger than their corresponding error bars. These variations could suggest density inhomogeneities along the nebula (from $\sim 2000$ to $\sim 2 \times 10^{4} \mathrm{~cm}^{-3}$ ).

\subsection{Atomic and Ionized Mass and Ionization Fraction}

We have estimated the total mass of atomic and ionized gas, $M_{\mathrm{H}}$ and $M_{\mathrm{H}^{+}}$, in the lobes of CRL 618 using the mean electron densities derived above and the total energy radiated by [O I] $\lambda \lambda 6300,6363$ and $\mathrm{H} \alpha$, respectively. The [O I] $(\mathrm{H} \alpha)$ intensity is proportional to the product of the electron density and the $\mathrm{H}\left(\mathrm{H}^{+}\right)$number density, assuming that the transitions are optically thin and that the electron tempera- 
ture does not strongly vary within the emitting region (Gurzadyan 1997; Osterbrock 1989). Considering a mean electron temperature in the shocked lobes of $10,000 \mathrm{~K}$, relative abundances of $\mathrm{He} / \mathrm{H}=0.1$ and $\mathrm{O} / \mathrm{H}=3.3 \times 10^{-4}$ (appropriate for a C-rich star like CRL 618; Lambert et al. 1986), and the required atomic parameters (see, e.g., Mendoza 1983; Gurzadyan 1997), we derive

$$
\begin{aligned}
M_{\mathrm{H}}^{[\mathrm{OI}]}\left(M_{\odot}\right)= & 1.3 \times 10^{-4}\left(\frac{n_{e}}{10^{4} \mathrm{~cm}^{-3}}\right)^{-1}\left(\frac{L_{[\mathrm{O} \mathrm{I}]}}{0.1 L_{\odot}}\right) \\
& \times\left(\frac{D}{900 \mathrm{pc}}\right)^{2}, \\
M_{\mathrm{H}^{+}}^{\mathrm{H} \alpha}\left(M_{\odot}\right)= & 9.6 \times 10^{-5}\left(\frac{n_{e}}{10^{4} \mathrm{~cm}^{-3}}\right)^{-1}\left(\frac{L_{\mathrm{H} \alpha}}{0.1 L_{\odot}}\right) \\
& \times\left(\frac{D}{900 \mathrm{pc}}\right)^{2},
\end{aligned}
$$

where $L$ is the dereddened luminosity of the line and $D$ the distance to the source. Note that equation (5) is valid for electron densities smaller than the critical density of [O $\mathrm{I}$, $n_{c} \sim 10^{6} \mathrm{~cm}^{-3}$, which is the case for CRL 618. On the other hand, this equation only provides a lower limit of the total atomic mass $\left(M_{\mathrm{H}}\right.$, which includes the $\mathrm{He}$ contribution), since we are assuming that most of the oxygen is neutral and in the ground state. In deriving equation (6) we have assumed the classic radiative recombination case $\mathrm{b}$ for $T_{e} \sim 10,000$ K (i.e., $z_{3}=n_{3} / n_{e} n_{\mathrm{H}^{+}}=0.25 \times 10^{-20} \mathrm{~cm}^{3}$, where $n_{3}$ is the population of the level $n=3$ of $\mathrm{H}$; Gurzadyan 1997).

We have separately estimated the masses of the east and west lobes using equations (5) and (6). For the east (west) lobe we have used an average electron density of $6.5 \times 10^{3}$ $\left(9 \times 10^{3}\right) \mathrm{cm}^{-3}$, an average extinction of $A_{V} \sim 2.7(3.1)$ mag, and the fluxes in Table 1 multiplied by (1) a factor of 2.5 , which converts the flux within a $1^{\prime \prime}$ wide slit to total nebular flux (estimated from the direct $H S T$ and ground-based images; Fig. 1), and (2) a factor of 0.5 and 0.8 , for the east and west lobe, respectively, that is the fraction of the total $\mathrm{H} \alpha$ luminosity locally produced in the lobes (not scattered emission; see $\S 5.1$ and Schmidt \& Cohen 1981). We note that point 2 applies only to the $\mathrm{H} \alpha$ luminosity and not to [O I] emission since the latter is not contaminated by scattered light. The masses derived for a distance to the source of $900 \mathrm{pc}$ are given in Table 4. In this table $M_{\mathrm{H}^{+}}^{\mathrm{H} \alpha}$ for the east lobe is the mass of ionized shocked gas plus the mass of ionized gas in region $\mathrm{A}^{\prime}$ (Fig. 1), since the latter contributes significantly to the total $\mathrm{H} \alpha$ luminosity. In contrast, $M_{\mathrm{H}}^{[\mathrm{OI}]}$ only accounts for the mass of atomic shocked gas, since region $\mathrm{A}^{\prime}$ is not detected in our [O I] long-slit spectra.

We have estimated the mass of ionized gas in region $\mathrm{A}^{\prime}$ from its $\mathrm{H} \alpha$ flux, which is $\sim 20 \%$ of the total flux (scattered

TABLE 4

ATOMIC AND IONIZED MASS IN CRL 618

\begin{tabular}{ccc}
\hline \hline Lobe & $\begin{array}{c}M_{\mathrm{H}}^{[\mathrm{O}]} \\
\left(M_{\odot}\right)\end{array}$ & $\begin{array}{c}M_{\mathrm{H}^{+}}^{\mathrm{H} \alpha} \\
\left(M_{\odot}\right)\end{array}$ \\
\hline East....... & $>1.3 \times 10^{-4}$ & $1.3 \times 10^{-4} \mathrm{a}$ \\
West...... & $>7.0 \times 10^{-5}$ & $4.0 \times 10^{-5}$ \\
\hline
\end{tabular}

a Mass of region $\mathrm{A}^{\prime}$ plus east lobe. plus local emission) of the east lobe. The electron density and extinction in this region are $\sim 10^{5} \mathrm{~cm}^{-3}$ and $A_{V}>6$, respectively ( $\S \S 5.2$ and 8 ). Using equation (6) we derive $M_{\mathrm{H}^{+}}^{\mathrm{A}^{\prime}}>4 \times 10^{-5} M_{\odot}$. The ionized mass in a spherical volume of gas with radius $0.5, n_{e} \sim 10^{5} \mathrm{~cm}^{-3}$, and ionization fraction $\sim 1$ (reasonable values for region $\mathrm{A}^{\prime}$ ) is $\sim 10^{-4} M_{\odot}$, also in agreement with our previous estimate.

Subtracting the ionized mass of region $\mathrm{A}^{\prime}$ from the total mass of the east lobe in Table 4 (second column), the mass of shock-ionized gas is (3-9) $\times 10^{-5} M_{\odot}$. In addition, for an east lobe-to-west lobe ionized mass ratio similar to the corresponding atomic mass ratio, which is 1.9 (first column in Table 4), $M_{\mathrm{H}^{+}}$for the east lobe would be $4 \times 10^{-5} M_{\odot}$, within the previous mass range. Note that a similar ionization fraction for the east and west lobes is suggested by the similar $[\mathrm{O} \mathrm{I}] / \mathrm{H} \alpha$ ratio in both lobes (without including the scattered component).

We have also estimated the mass of ionized material in CRL 618 geometrically, using the mean electron densities and the volume of the shocked lobes directly measured from the HST images, assuming a cylindrical geometry for the lobe components. We have separately considered the case of hollow lobes (with 0 ". 1 thick walls) and lobes uniformly filled with gas. Assuming that the majority of the electrons in the nebula come from $\mathrm{H}^{+}$, i.e., $n_{e} \approx n_{\mathrm{H}^{+}}$, the ionized mass of the west and east lobes (excluding region $\mathrm{A}^{\prime}$ ) for hollow (full) cylinders is $3.5 \times 10^{-5}\left(1.1 \times 10^{-4}\right)$ and $4.2 \times 10^{-5}$ $\left(1.3 \times 10^{-4}\right) M_{\odot}$, respectively. These values are consistent with those previously obtained from the $\mathrm{H} \alpha$ luminosity. The hollow-cylinder geometry is a better approximation to the structure of the lobes of CRL 618 than the filled-cylinder geometry: for the latter, the derived masses systematically exceed those obtained from the $\mathrm{H} \alpha$ luminosity (Table 4).

The high intensity of the [O $\mathrm{I}]$ lines relative to $\mathrm{H} \alpha$ and, in general, the observed ratios for the rest of the lines suggest a low ionization fraction, $X=n_{e} / n_{\mathrm{H}} \sim 0.03-0.1$, in the lobes, by comparison with the predictions of planar shock models by Hartigan et al. (1994). These values are also consistent with the upper limits to $X$ derived from the ionized and atomic masses calculated above $\left(X \approx 1.4 M_{\mathrm{H}^{+}} / M_{\mathrm{H}}\right)$, $X<0.8$ and $X<0.6$ for the west and east lobe, respectively. An ionization fraction of only a few percent seems to be inconsistent with the high velocity of the shocks in CRL $618\left(\sim 200 \mathrm{~km} \mathrm{~s}^{-1}\right)$ deduced from the line width: full ionization is expected for planar shocks with $V_{s} \gtrsim 80 \mathrm{~km} \mathrm{~s}^{-1}$ (Hartigan et al. 1994). The fact that planar shocks are not appropriate for CRL 618 could partially (but probably not totally) resolve this problem. Fast bow shocks produce a range of ionization fractions along the bow shock, with a large $X$-value at the head of the shock but smaller at the wings, where the normal component of the shock velocity is substantially smaller. The result is a smaller mean ionization fraction than that predicted by planar shocks moving at the same velocity, which produces equally high $X$-values all along the front.

\section{DISCUSSION}

\subsection{Formation and Evolution of the Circumstellar Envelope of CRL 618}

It is widely accepted that the actual nebular geometry and kinematics of CRL 618 are the result of the interaction between the circumstellar material mostly resulting from 
the AGB mass-loss process and fast stellar winds ejected more recently, probably in the post-AGB phase ( $(1)$. Under this scenario the optical shock-excited, rapidly expanding lobes are made of circumstellar material that is undergoing the passage of shock fronts produced by the wind interaction.

Our results support this scenario and add more details on how this process is taking place. In our opinion, the morphology of the nebula, consisting of several multiply directed narrow lobes, suggests that the post-AGB wind is collimated rather than spherical. The presence of lobe components directed along different axes indicates multiple ejection events that could have happened with different orientations and velocities and/or at different epochs. The bright ripple-like structure found along the lobes could be due to instabilities in the flow or bow shocks resulting from the episodic interaction between fast collimated jets and the slowly expanding envelope ejected during the AGB phase (see a more detailed analysis of and discussion on HST imaging in different filters in R. Sahai et al. 2002, in preparation).

The complex spatiokinematical structure of the lobes leads to kinematical ages ranging from $\sim 100 \mathrm{yr}$ in the innermost regions of the lobes to $\sim 400 \mathrm{yr}$ at the tips, without correcting for projection effects. This age difference could be real, in which case the innermost regions would have been more recently shocked, or it could be due to different inclinations of, and/or different decelerations suffered by, the lobe subcomponents. Adopting a mean inclination of $24^{\circ}$, the age of the optical nebula is $\lesssim 180 \mathrm{yr}$.

The electron density does not vary systematically along the shocked lobes of CRL 618 ( $\$$ 9.1). Since the ionization fraction is not expected to vary drastically along the lobes (note that $V_{s}$, given by the line FWZI, and the $[\mathrm{O} \mathrm{I}] / \mathrm{H} \alpha$ line ratio have similar values all along the nebula), a roughly constant $n_{e}$ suggests that the total density is constant as well. Such a density distribution is most likely inconsistent with most of the [S II] emission (and, probably, most forbidden lines) arising from the lobe interior, which is presumably filled with the fast post-AGB wind whose density is expected to decrease with the distance (C.-F. Lee \& R. Sahai 2002, in preparation). We think that most of the emission arises from shocked gas in thin-walled lobes, a conclusion supported by the limb-brightened appearance of the lobes in the HST images (Fig. 1) and the better agreement between the geometrical and $\mathrm{H} \alpha$ masses of ionized gas derived in $\S 9.2$.

The spatial distribution of the radial optical thickness beyond the tips of the lobes suggests a high contrast between the density of dust in the gas filling the lobes and that in front of them, in the AGB CSE. This is consistent with the optical lobes of CRL 618 being cavities excavated in the AGB envelope by the impact of the post-AGB wind (but see $\S 8.2$ for caveats).

The forward shocks accelerating and shaping the lobes of CRL 618 are very likely in a radiative regime because the cooling time of the gas immediately behind the shock front in the lobes, $t_{\text {cool }}$, is significantly smaller than the dynamical timescale of the nebula ( $\lesssim 180 \mathrm{yr}$ ). Immediately after the shock, the gas temperature is expected to rise to $\sim 10^{5}-10^{6} \mathrm{~K}$ for a shock speed of $\sim 200 \mathrm{~km} \mathrm{~s}^{-1}$ (e.g., Dalgarno \& McCray 1972). The time required by the gas to cool down to the present temperature of the shocked optically emitting lobes (of the order of $10^{4} \mathrm{~K}$ ) is $t_{\text {cool }} \propto V_{s}^{3} / n_{0} \Lambda(T)$, where $V_{s}$ is the shock velocity, $n_{0}$ is the preshock number density of nuclei, and $\Lambda(T)$ is the cooling rate (Blondin, Fryxell, \& Konigl 1990). For a shock velocity of $V_{s} \lesssim 200 \mathrm{~km} \mathrm{~s}^{-1}, \Lambda(T)=$ few times $10^{-22}$ ergs $\mathrm{cm}^{-3} \mathrm{~s}^{-1}$ is appropriate (Dalgarno \& McCray 1972) and $t_{\text {cool }}(\mathrm{yr}) \approx 570 V_{s, 7}^{3} / n_{0}$, where $V_{s}$ and $n_{0}$ are given in units of $10^{7} \mathrm{~cm} \mathrm{~s}^{-1}$ and $\mathrm{cm}^{-3}$, respectively. The density of the molecular AGB envelope at a radial distance of $\sim 7^{\prime \prime}$, i.e., ahead of the shocked lobes, provides a lower limit to the density of the preshock material since the first interactions between the post-AGB wind and the AGB CSE presumably took place closer to the central star. For a massloss rate $M=5 \times 10^{-5} M_{\odot} \mathrm{yr}^{-1}$ and expansion velocity $17.5 \mathrm{~km} \mathrm{~s}^{-1}(\S 1), n_{\mathrm{H}_{2}}=6 \times 10^{4} \mathrm{~cm}^{-3}\left(n_{0} \sim 2 n_{\mathrm{H}_{2}}\right.$, assuming atomic preshock gas). For the numbers above, we derive $t_{\text {cool }}<0.04$ yr. Even accounting for an uncertainty in the preshock density of 1 order of magnitude, the cooling time is much smaller than the dynamical timescale.

Thin lobe walls, where most of the shocked material seems to be located, and high electron densities, up to $\sim 10^{4}$ $\mathrm{cm}^{-3}$, are both expected in the case of radiative shocks: the low thermal pressure after the shock passage leads to the collapse of the shocked material in a very small and dense region after the shock (for a review see, e.g., Frank 1999). The large length-to-width ratio of the lobes, $\sim 7$, is also in better agreement with the shocks being radiative, since for the adiabatic case the thermal pressure of the shocked gas is very high, leading to inflated bubble-shaped lobes.

The nature of the reverse shocks, propagating in the postAGB wind itself toward the nebula center, is uncertain because it depends on the unknown physical properties of the post-AGB wind. For $\rho_{\mathrm{AGB}} / \rho_{\mathrm{pAGB}} \sim 600(\S 8.2)$ and a reverse shock velocity of the order of $100 \mathrm{~km} \mathrm{~s}^{-1}$, the cooling time of the shocked post-AGB gas would be $\lesssim 12 \mathrm{yr}$, and therefore the reverse shocks would also be in a radiative regime.

In the absence of a presently active energy input, the dense gas in the optical lobes of CRL 618 should be at a temperature significantly less than $10^{4} \mathrm{~K}$. The high electron densities in the lobes of CRL 618 would have led to a very quick recombination of the presently shocked and ionized gas: the recombination timescale is about $10 \mathrm{yr}$ for a total hydrogen recombination coefficient $\alpha_{\mathrm{H}}\left(10^{4} \mathrm{~K}\right)=4.18 \times 10^{-13} \mathrm{~cm}^{-3}$ $\mathrm{s}^{-1}$ (see, e.g., Kwok 2000 and references therein). Moreover, the time for the shocked gas to cool down 1 order of magnitude (to $\sim 1000 \mathrm{~K}$ ) is $\lesssim 2 \mathrm{yr}$, using a cooling rate $\Lambda \gtrsim 10^{-25}$ ergs $\mathrm{cm}^{-3} \mathrm{~s}^{-1}$, appropriate for $T_{e} \sim 10^{4} \mathrm{~K}$ and ionization fraction $\gtrsim 0.01$ (Dalgarno \& McCray 1972). Such a short cooling time implies that, in the absence of a continuous ionization/heating source, the gas in the lobes of CRL 618 should have become atomic and cool in a timescale much smaller than the age of the nebula, $\$ 180 \mathrm{yr}$. Even if we have overestimated its kinematical age, CRL 618 is at least about 30 years old, since it was observed with similar characteristics in 1973 by, e.g., Westbrook et al. (1975). The most likely energy source for maintaining a high temperature $(>1000$ $\mathrm{K})$ in the lobes is an ongoing interaction between the postAGB wind and the circumstellar material (note that the central star is not hot enough to ionize and heat the lobes). This implies that the fast wind, which is ultimately responsible for the shaping and evolution of its CSE, is currently active in CRL 618.

This fast wind is not necessarily stationary but may rather be pulsed (episodic) with a period smaller but comparable to the recombination timescale of the shocked gas $(\sim 10 \mathrm{yr})$. 
In this case, the shocked material (initially fully ionized) would have time to partially recombine between pulses, which might be a contributing factor to maintaining a low ionization fraction in the lobes of CRL 618 in spite of the high velocity of the shocks. On the other hand, to keep the temperature of the shocked gas above $T_{e}>10^{3} \mathrm{~K}$, the pulse period should be greater than $2 \mathrm{yr}$. The idea of a pulsating fast wind is consistent with the presence of the multiple bright, bow-shaped and ripple-like structures seen along the lobes of CRL 618 (Fig. 1) since their separations correspond to timescales of $\sim 10 \mathrm{yr}$.

Finally, we have reported a recent increase (decrease) of the intensity of the [O III] $\lambda 5007$ ([O I] $\lambda 6300$ ) lines in CRL 618 ( $\S 4.2$ ). This change also provides indirect evidence for the presence of continuously evolving shocks resulting from a continuous interaction between the ongoing post-AGB wind and the surrounding media. Since shocks are the main excitation mechanism of the gas in the lobes of CRL 618, where most of the forbidden line emission is detected, the observed [O III] brightening probably has its origin in a change of the shock properties (rather than in the stellar radiation). The $[\mathrm{O} \mathrm{III}] / \mathrm{H} \beta$ ratio is very sensitive to the shock velocity (HRH87), and therefore the recent increase of the [O III] flux may indicate that the ongoing shocks are faster now than 10 years ago. As a consequence of the shock acceleration, the ionization fraction of the lobes could have increased, leading to a weakening of the [O I] lines. The increasing velocity of the shocks could point to acceleration of the stellar post-AGB wind. The timescale of the [O III]/ $[\mathrm{O}$ I] brightening/weakening (observed over $\gtrsim 10 \mathrm{yr}$ ) is consistent with the expected shock velocity and the size of the shocked-gas emitting region: a $90-200 \mathrm{~km} \mathrm{~s}^{-1}$ shock will cross the 0 ". 1 wide lobe walls $\left(\sim 1.3 \times 10^{15} \mathrm{~cm}\right)$ in only $4.7-$ $2.1 \mathrm{yr}$.

\subsection{Future Circumstellar Evolution of CRL 618}

The shocked optical lobes of CRL 618 could evolve into a fast, bipolar molecular outflow if the timescale of molecular reformation in the shocked gas is smaller than the time required by the expanding ionization front (produced by the central star) to reach and fully ionize the whole nebula.

The present radiative, forward shocks will continue propagating through the molecular cocoon (traced by $\mathrm{CO}$ ), accelerating and shaping more and more material of the AGB CSE. We are observing the beginning of this process, when the shocked material is still hot (and, therefore, primarily visible in the optical) and its mass is still low compared to that of the unshocked envelope. The hot shocked material will cool down, allowing molecule reformation, and would be observed as an extended, bipolar outflow moving at high velocity. This is what presumably already happened to a part of the shocked material: the fast molecular bipolar outflow observed in the innermost parts of CRL 618 ( $\S 1$, discussed in detail in C. Sánchez Contreras et al. 2002, in preparation). If the shocks have sufficient energy to accelerate most of the AGB envelope, the mass of the future fast, bipolar outflow of CRL 618 could be a significant fraction of the total mass of the AGB CSE, $\sim 1.5 M_{\odot}$. The interaction of this fast and massive molecular jet with the interstellar medium or the low-density, outermost parts of the CRL 618 CSE could also lead to new shocks that would be observed, in their first stages, through optical line emission surrounding the molecular bipolar outflow.
In our opinion, the possible future evolution of CRL 618 described above is what already occurred in another wellstudied PPN, OH 231.8+4.2. This object shows a very collimated and fast molecular outflow that was presumably shaped and accelerated by radiative shocks resulting from a two-wind interaction process $\sim 1000$ years ago (Sánchez Contreras et al. 2000; Alcolea et al. 2001); this outflow should have been in the past a very intense emitter of recombination and forbidden lines at short wavelengths, as the optical lobes in CRL 618. Surrounding the collimated molecular outflow of $\mathrm{OH} 231.8+4.2$, there is a wide bowshaped nebula of shock-excited warm gas that is observed in the visible (Bujarrabal et al. 2002; Sánchez Contreras et al. 2000). This component is believed to result from the hydrodynamical interaction between the bipolar $\mathrm{CO}$ outflow and the tenuous circumstellar material, the shocks generated in this interaction being more likely in an adiabatic regime.

The central star of $\mathrm{OH} 231.8+4.2$ is still very cool $(\sim 2500$ $\mathrm{K}$ ) and therefore is not able to ionize the circumstellar material. The evolution of the optical lobes of CRL 618 into a fast molecular outflow (like in $\mathrm{OH} 231.8+4.2$ ) in the future requires that, once the wind interaction stops, the time required by the ionization front, produced by the hot central star, to reach the lobes $\left(t_{\text {ion }}\right)$ be larger than the molecular reformation timescale $\left(t_{\mathrm{mol}}\right)$.

In the relatively dense envelopes of PPNs, the timescale for molecule formation is $\lesssim 1000 \mathrm{yr}$, as deduced from the widespread existence of fast $\left(V \gtrsim 100 \mathrm{~km} \mathrm{~s}^{-1}\right.$ ) molecular outflows with kinematical ages $\lesssim 10^{3}$ yr (see, e.g., Bujarrabal et al. 2001); note that molecules are totally destroyed by shocks at speeds $\gtrsim 30 \mathrm{~km} \mathrm{~s}^{-1}$ (Hollenbach \& McKee 1989), and therefore molecules moving at such high velocities have most likely been reformed in the postshock gas. The kinematical age of the compact molecular outflow of CRL 618 itself, moving at $\gtrsim 100 \mathrm{~km} \mathrm{~s}^{-1}$, is only of the order of $10^{2} \mathrm{yr}(\S 1)$, so, in general, we can expect molecule reformation to occur in $t_{\mathrm{mol}} \sim 10^{2}-10^{3} \mathrm{yr}$. This value is also in reasonable agreement with theoretical estimates of $t_{\mathrm{mol}}$ in dense, $n_{\mathrm{H}} \sim 10^{6} \mathrm{~cm}^{-3}$, postshock gas (Hollenbach \& McKee 1989).

The time required by the ionization front to reach the lobes, $t_{\text {ion }}$, depends on the relative expansion velocity of the front and the lobes. The expansion velocity of the ionization front in CRL 618, estimated from radio continuum mapping with high angular resolution in different epochs, has decreased with time: from 50-74( $D / 0.9 \mathrm{kpc}$ ) (Kwok \& Feldman 1981) to $26(D / 0.9 \mathrm{kpc}) \mathrm{km} \mathrm{s}^{-1}$ (Spergel, Giuliani, \& Knapp 1983). Expanding at this rate, the ionization front will never reach the lobes, which are moving much faster (up to $200 \mathrm{~km} \mathrm{~s}^{-1}$ ), and the molecules will reform in the shocked gas sooner or later. If the shocked lobes are decelerated by interaction with the surrounding circumstellar gas and/or the velocity of the ionization front increases (as a result, e.g., of the evolution of the central star, which will become hotter and hotter, and the dilution of the circumstellar gas), then the UV stellar photons will be able to fully ionize the lobes of CRL 618. The ionization timescale depends on different unknown physical parameters of the central star, such as mass, temperature, mass-loss rate, etc., and their evolution with time. The shocked gas would be observed as a molecular outflow during $t_{\text {ion }}-t_{\text {mol }}$, whenever $t_{\text {ion }}>t_{\text {mol }}$, otherwise CRL 618 will never develop a massive bipolar molecular outflow (like, e.g., $\mathrm{OH}$ $231.8+4.2$ ). 


\section{CONCLUSIONS}

We have obtained ground-based, long-slit spectra in the ranges 6233-6806 and 4558-5331 $\AA$ for three different ( $1^{\prime \prime}$ wide) slit positions in the PPN CRL 618. Based on the analysis of these spectra and direct ( $\mathrm{H} \alpha$ and continuum) images, we find the following results:

1. The Balmer line emission results from the superposition of unscattered and scattered components. The unscattered emission is locally produced in the shocked lobes and in an inner, compact region labeled $A^{\prime}$ (Fig. 1). The scattered radiation originates in the central $\mathrm{H}$ II region (and also maybe in region $\mathrm{A}^{\prime}$ ) and is reflected by the dust in the shocked lobes and in the unshocked AGB envelope. Most forbidden lines are entirely produced in the lobes, but there is also forbidden line emission (e.g., [Fe III] and [S III]) arising in the $\mathrm{H}$ II region and region $\mathrm{A}^{\prime}$.

2. We identify the W-R bump at $\sim 6540 \AA$ superimposed on the scattered continuum. This bump and a number of high-excitation transitions, such as $\mathrm{He}$ II $\lambda 4686$, originate in the dense $\left(n_{e} \sim 10^{5}-10^{7} \mathrm{~cm}^{-3}\right)$ inner $\mathrm{H}$ II region and are seen after being reflected by the nebular dust.

3. The spectrum of region $A^{\prime}$ is similar to that of the inner $\mathrm{H}$ II region (dominated by high-excitation lines) but remarkably different from the spectrum of the shocked lobes (dominated by low-excitation lines) and is consistent with $n_{e} \sim 10^{4}-10^{5} \mathrm{~cm}^{-3}$ and full ionization in this region. Thus, region $\mathrm{A}^{\prime}$ very likely represents the outermost parts of the central H II region; i.e., it is gas ionized by the UV stellar radiation rather than by shocks.

4. The shock velocity derived from the profiles of line emission arising in the shocked lobes is $\gtrsim 200 \mathrm{~km} \mathrm{~s}^{-1}$. However, the line ratios in the lobes are consistent with the predictions of bow shock models with a lower shock velocity (less than about $90 \mathrm{~km} \mathrm{~s}^{-1}$, and probably $\sim 75 \mathrm{~km} \mathrm{~s}^{-1}$ ).

5. We have measured an increase (decrease) of the [O III] $\lambda 5007$ ([O I] $\lambda 6300$ ) line flux by a factor of $\sim 2.5(0.7)$ with respect to observations performed $\sim 10$ years ago. Such variations could be due to a recent increase of the shock velocity.

6. The mean nebular inclination (with respect to the plane of the sky) is about $24^{\circ}$ and, very likely, less than $39^{\circ}$.

7. The material in the lobes of CRL 618 is moving away from the star at velocities up to $\sim 200 \mathrm{~km} \mathrm{~s}^{-1}$. The projected velocity of the gas shows a nonlinear radial variation, resulting in a kinematical age that varies from $\sim 100 \mathrm{yr}$ near the nebular center to $\sim 400 \mathrm{yr}$ at the tips, without correcting projection effects. For a mean inclination of $i=24^{\circ}$, the kinematical age of the optical lobes is $\lesssim 180 \mathrm{yr}$.

8. The inner $\mathrm{H}$ II region and region $\mathrm{A}^{\prime}$ seem to be expanding at moderate velocity, $\lesssim 30 \mathrm{~km} \mathrm{~s}^{-1}$, much slower than the shocked lobes.
9. The extinction along the nebula is found to decrease from the center $\left(A_{V}>10 \mathrm{mag}\right)$ to the lobe tips $(\sim 2 \mathrm{mag})$; beyond the bright optical lobes, where an $\mathrm{H} \alpha$ scattered halo is visible, the extinction increases again. This increase indicates a large density contrast between the dust inside the optical lobes and beyond them, i.e., in the AGB CSE unaltered by the shocks. This result is consistent with the lobes of CRL 618 being cavities in the AGB CSE excavated by post-AGB winds.

10. The electron density, with mean values $n_{e} \sim(5-8) \times 10^{3}$ and $\sim 8 \times 10^{3}-10^{4} \mathrm{~cm}^{-3}$, for the east and west lobe, respectively, shows no systematic variation along the lobes.

11. The ionization fraction in the lobes is $X \sim 0.03-0.1$, derived by comparison of different line ratios with predictions from planar shock models. The total number density in the lobes is then $\sim 10^{5}-10^{6} \mathrm{~cm}^{-3}$.

12. The mass of atomic gas in the east (west) lobe is $>1.3 \times 10^{-4}\left(>7.0 \times 10^{-5}\right) M_{\odot}$. The masses of ionized gas in the lobes are smaller: $\sim 6 \times 10^{-5} M_{\odot}$ for the east lobe and $\sim 4 \times 10^{-5} M_{\odot}$ for the west lobe. The ionized mass in region $\mathrm{A}^{\prime}$ is comparable to the ionized mass in the lobes or even higher, up to $\sim 10^{-4} M_{\odot}$.

13. The limb brightening of the optical lobes and the lack of a systematic spatial gradient in $n_{e}$ suggest that most of the line emission arises in a thin shell of shocked material rather than in gas filling the interior of the lobes. The thin-shell geometry is expected from radiative shocks shaping and accelerating the AGB CSE. The radiative nature of the shocks is inferred from the short cooling time of the immediate postshock gas, $\lesssim 0.04 \mathrm{yr}$ (relative to the dynamical time).

14. The post-AGB wind is probably currently active, shocking and heating the AGB circumstellar material, since the time required for the gas in the shocked lobes (presently partially ionized) to cool down significantly below $10^{4} \mathrm{~K}$ is $\lesssim 2$ yr, much shorter than the age of the nebula.

The authors are grateful to Noam Soker for reading and commenting on this paper, to A. Castro-Carrizo for helping during the long-slit observations, and to C.-F. Lee for fruitful conversations during the writing of this paper. This work was performed at the Jet Propulsion Laboratory, California Institute of Technology, under a contract with the National Aeronautics and Space Administration, and has been partially supported by a NASA Long Term Space Astrophysics grant to R. S. This research has made use of the USNOFS Image and Catalog Archive operated by the United States Naval Observatory, Flagstaff Station (http://www.nofs. navy.mil/data/fchpix). The authors also acknowledge the use of NASA's Astrophysical Data System Abstract Service (ADS).

\section{REFERENCES}

Alcolea, J., Bujarrabal, V., Sánchez Contreras, C., Neri, R., \& Zweigle, J. 2001, A\&A, 373, 932

Aller, L. H., \& Keyes, C. D. 1987, ApJS, 65, 405

Bachiller, R., Gómez-González, J., Bujarrabal, V., \& Martín-Pintado, J. 1988, A\&A, 196, L5

Baessgen, M., Hopfensitz, W., \& Zweigle, J. 1997, A\&A, 325, 277

Blondin, J. M., Fryxell, B. A., \& Konigl, A. 1990, ApJ, 360, 370

Bujarrabal, V., Alcolea, J., Sánchez Contreras, C., \& Sahai, R. 2002, A\&A, 389, 271

Bujarrabal, V., Castro-Carrizo, A., Alcolea, J., \& Sánchez Contreras, C. 2001, A\&A, 377, 868

Bujarrabal, V., Gomez-Gonzalez, J., Bachiller, R., \& Martín-Pintado, J. 1988, A\&A, 204, 242

Cantó, J., Meaburn, J., Theokas, A. C., \& Elliott, K. H. 1980, MNRAS, 193, 911

Cardelli, J. A., Clayton, G. C., \& Mathis, J. S. 1989, ApJ, 345, 245

Carsenty, U., \& Solf, J. 1982, A\&A, 106, 307 (CS82)

Cernicharo, J., Guélin, M., Martín-Pintado, J., Peñalver, J., \& Mauersberger, R. 1989, A\&A, 222, L1

Dalgarno, A., \& McCray, R. A. 1972, ARA\&A, 10, 375

Frank, A. 1999, NewA Rev., 43, 31

Gammie, C. F., Knapp, G. R., Young, K., Phillips, T. G., \& Falgarone, E. 1989, ApJ, 345, L87

Goodrich, R. W. 1991, ApJ, 376, 654

Gottlieb, E. W., \& Liller, W. 1976, ApJ, 207, L135 
Gurzadyan, G. A. 1997, The Physics and Dynamics of Planetary Nebulae (Berlin: Springer)

Hajian, A. R., Phillips, J. A., \& Terzian, Y. 1996, ApJ, 467, 341

Hartigan, P., Morse, J. A., \& Raymond, J. 1994, ApJ, 436, 125

Hartigan, P., Raymond, J., \& Hartmann, L. 1987, ApJ, 316, 323 (HRH87)

Hollenbach, D., \& McKee, C. F. 1989, ApJ, 342, 306

Howarth, J. D. 1983, MNRAS, 203, 301

Kaler, J. B. 1978, ApJ, 220, 887

Kastner, J., Soker, N., \& Rappaport, S. A., eds. 2000, ASP Conf. Ser. 199, Asymmetrical Planetary Nebulae II: From Origins to Microstructures (San Francisco: ASP)

Kelly, D. M., Latter, W. B., \& Rieke, G. H. 1992, ApJ, 395, 174

Knapp, G. R., \& Morris, M. 1985, ApJ, 292, 640

Knapp, G. R., Sandell, G., \& Robson, E. I. 1993, ApJS, 88, 173

Kwok, S. 2000, The Origin and Evolution of Planetary Nebulae (Cambridge: Cambridge Univ. Press)

Kwok, S., \& Bignell, R. C. 1984, ApJ, 276, 544

Kwok, S., \& Feldman, P. A. 1981, ApJ, 247, L67

Lambert, D. L., Gustafsson, B., Eriksson, K., \& Hinkle, K. H. 1986, ApJS, 62,373

Leuenhagen, U., Hamann, W.-R., \& Jeffery, C. S. 1996, A\&A, 312, 167

Martín-Pintado, J., Bujarrabal, V., Bachiller, R., Gómez-González, J., \& Planesas, P. 1988, A\&A, 197, L15

Martín-Pintado, J., Gaume, R., Bachiller, R., \& Johnson, K. 1993, ApJ, 419,725

Meixner, M., Campbell, M. T., Welch, W. J., \& Likkel, L. 1998, ApJ, 509, 392
Mendoza, C. 1983, in IAU Symp. 103, Planetary Nebulae, ed. D. R. Flower (San Francisco: ASP), 143

Neri, R., García-Burillo, S., Guèlin, M., Guilloteau, S., \& Lucas, R. 1992, A\&A, 262, 544

Osterbrock, D. E. 1989, Astrophysics of Gaseous Nebulae and Active Galactic Nuclei (Mill Valley: University Science Books)

Phillips, J. P., Williams, P. G., Mampaso, A., \& Ukita, N. 1992, A\&A, 260, 283

Reipurth, B., Yu, K., Heathcote, S., Bally, J., \& Rodríguez, L. F. 2000, AJ, 120,1449

Riera, A., Phillips, J. P., \& Mampaso, A. 1990, Ap\&SS, 171, 231

Sahai, R., \& Trauger, J. T. 1998, AJ, 116, 1357

Sánchez Contreras, C., Bujarrabal, V., Miranda, L. F., \& FernándezFigueroa, M. J. 2000, A\&A, 355, 1103

Schmidt, G. D., \& Cohen, M. 1981, ApJ, 246, 444

Schwarz, H. E., Aspin, C., Corradi, R. L. M., \& Reipurth, B. 1997, A\&A, 319,267

Spergel, D. N., Giuliani, J. L., \& Knapp, G. R. 1983, ApJ, 275, 330

Trammell, S. R. 2000, in ASP Conf. Ser. 199, Asymmetrical Planetary Nebulae II: From Origins to Microstructures, ed. J. Kastner, N. Soker, \& S. A. Rappaport (San Francisco: ASP), 147

Trammell, S. R.. Dinerstein, H. L., \& Goodrich, R. W. 1993, ApJ, 402, 249

Tylenda, R., Acker, A., \& Stenholm, B. 1993, A\&AS, 102, 595

Welch, C. A., Frank, A., Pipher, J. L., Forrest, W. J., \& Woodward, C. E. 1999, ApJ, 522, L69

Westbrook, W. E., Becklin, E. E., Merrill, K. M., Neugebauer, G. Schmidt, M., Willner, S. P., \& Wynn-Williams, C. G. 1975, ApJ, 202, 407 